\title{
LÉVY-KHINTCHINE DECOMPOSITIONS FOR GENERATING FUNCTIONALS ON ALGEBRAS ASSOCIATED TO UNIVERSAL COMPACT QUANTUM GROUPS
}

\author{
BISWARUP DAS, UWE FRANZ, ANNA KULA, AND ADAM SKALSKI
}

\begin{abstract}
We study the first and second cohomology groups of the ${ }^{*}$-algebras of the universal unitary and orthogonal quantum groups $U_{F}^{+}$and $O_{F}^{+}$. This provides valuable information for constructing and classifying Lévy processes on these quantum groups, as pointed out by Schürmann. In the case when all eigenvalues of $F^{*} F$ are distinct, we show that these *-algebras have the properties (GC), (NC), and (LK) introduced by Schürmann and studied recently by Franz, Gerhold and Thom. In the degenerate case $F=I_{d}$, we show that they do not have any of these properties. We also compute the second cohomology group of $U_{d}^{+}$ with trivial coefficients $-H^{2}\left(U_{d}^{+}, \mathbb{C}_{\epsilon}\right) \cong \mathbb{C}^{d^{2}-1}$ - and construct an explicit basis for the corresponding second cohomology group for $O_{d}^{+}$(whose dimension was known earlier thanks to the work of Collins, Härtel and Thom).
\end{abstract}

\section{INTRODUCTION}

The theory of Lévy processes on involutive bialgebras subsumes both the theory of Lévy processes with values in topological groups and the theory of factorizable representations of current groups and algebras. The first of these two theories describes stochastic processes with independent and stationary increments. Its paradigms are Brownian motion and the Poisson process and it played a central role in the invention of classical stochastic calculus, see $\mathrm{App}_{1}, \mathrm{App}_{2}$ and the references therein. The second was motivated by quantum field theory [Ara] and was instrumental in the development of quantum stochastic calculus, cf. Acc, Str].

Noncommutative Lévy processes (initially called "white noises") were first introduced on $\mathbb{Z}_{2}$-graded involutive bialgebras $[\mathrm{ASvW}]$ and later extended to arbitrary graded (or braided) involutive bialgebras $\mathrm{Sch}_{2}$ and dual groups $\mathrm{Sch}_{3}$. It was soon realized that cohomology plays an important role in classifying and constructing Lévy processes on a given *-bialgebra, see $\left[\mathrm{Sch}_{1}, \mathrm{Sch}_{2}\right.$ and also $\left.\mathrm{FGT}, \mathrm{BFG}\right]$ for recent progress in this direction.

Based on Schürmann's work $\mathrm{Sch}_{2}$, the standard approach to classifying Lévy processes on a given involutive bialgebra or dual group consists in classifying first its ${ }^{*}$-representations acting on a pre-Hilbert space and then determining their first cohomology group as well as the second cohomology group with trivial coefficients. For the Woronowicz quantum group $S U_{q}(2)$ this programme was carried out by Schürmann and Skeide in the nineties [ScS, Ske 1 . Knowing the Lévy processes on some quantum group gives geometric information on its structure and can be used to construct Dirac operators [CFK].

2010 Mathematics Subject Classification. Primary 16T20, Secondary 16T05.

Key words and phrases. Hopf *-algebra; cocycle; generating functional; cohomology groups; quantum Lévy process; quantum group. 
If the $\mathrm{C}^{*}$-algebra associated to a given compact quantum group is of type $\mathrm{I}$, as it happens for $S U_{q}(2)$, then all *-representations of the $\mathrm{C}^{*}$-algebra are direct integrals of irreducible ones. In this case, if the irreducible ${ }^{*}$-representations are known, it can be feasible to use the approach outlined above to classify all Lévy processes on a given involutive bialgebra.

But there exist many interesting compact quantum groups whose $\mathrm{C}^{*}$-algebras are not of type I and whose representation theory is too wild to be classified. This is the case of the universal unitary $U_{F}^{+}$and the universal orthogonal $O_{F}^{+}$quantum groups (see Examples 1.2 and 1.5 for definitions). Then one can still gain a better understanding by studying the second cohomology group with trivial coefficients and the first cohomology group of some special *-representations, as was done for quantum permutation groups in [FKS. In this paper we start from Schürmann's work $\mathrm{Sch}_{1}, \mathrm{Sch}_{2}$ on the Brown-Glockner-von Waldenfels algebra Bro, GvW], i.e. the ${ }^{*}$-algebra generated by the coefficients of a $d \times d$ unitary matrix with noncommuting entries, see Example 1.1, and use his results to study the universal unitary and orthogonal quantum groups $U_{F}^{+}$and $O_{F}^{+}$.

In our study we consider mainly the following two extremal cases: the so-called generic case, that is the case where the eigenvalues of $F^{*} F$ are pairwise distinct, see Section 2, and the maximally degenerate case where $F=I_{d}$, see Section 3 . In the generic case we show that all Gaussian cocycles on *-algebras of the compact quantum groups $U_{F}^{+}$and $O_{F}^{+}$admit a generating functional and can therefore be used to construct Lévy processes on these quantum groups, see Theorems 2.1 and 2.2. Using the terminology of [FGT], see also Definition [1.9, this means that these *algebras have the property (GC). This implies that all their generating functionals admit a Lévy-Khintchine-type decomposition into a Gaussian part and a purely non-Gaussian part. Using similar methods, we show that the *algebra associated to the compact quantum group $S U_{q}(3)$ introduced by Woronowicz $\mathrm{Wo}_{2}$ does not have property (GC), see Proposition 2.3. This allows us to conclude that the (GC) property need not pass neither from a quantum group to its quantum subgroup, nor from a quantum subgroup to the whole quantum group.

In Section 3 we consider the maximally degenerate case $F=I_{d}$ and classify the cocycles of the one-dimensional representations of the ${ }^{*}$-algebras of the compact quantum groups $U_{d}^{+}:=U_{I_{d}}^{+}$and $O_{d}^{+}:=O_{I_{d}}^{+}$. We also give necessary and sufficient conditions for these cocycles to admit a generating functional and therefore a Lévy process. It follows from these results that the ${ }^{*}$-algebras of the compact quantum groups $U_{d}^{+}$with $d \geq 2$ and $O_{d}^{+}$with $d \geq 3$ have none of the properties (GC), (NC), or (LK), see Theorems 3.5 and 3.10, This provides the first examples of quantum groups which are non-cocommutative and do not have property $(\mathrm{LK})$, and also has certain consequences for general non-generic $U_{F}^{+}$and $O_{F}^{+}$.

The last section of this paper contains computations of the second cohomology group with trivial coefficients of these ${ }^{*}$-algebras. Collins, Härtel, and Thom [CHT] (see also Bic ]) have shown that the second cohomology group with trivial coefficients of the ${ }^{*}$-algebra of $O_{d}^{+}$has dimension $\frac{d(d-1)}{2}$. Here we show that $H^{2}\left(U_{d}^{+},{ }_{\epsilon} \mathbb{C}_{\epsilon}\right)$, the second cohomology group of the ${ }^{*}$-algebra of $U_{d}^{+}$with trivial coefficients, has dimension $d^{2}-1$ (Theorem 4.6) and describe an explicit basis for it. For that, we define a 'defect map' which measures how far a cocycle is from being a coboundary, and show that this map induces an isomorphism between $H^{2}\left(U_{d}^{+},{ }_{\epsilon} \mathbb{C}_{\epsilon}\right)$ and $s l(d, \mathbb{C})$, the space of complex $d \times d$ matrices with trace zero. A similar method establishes an isomorphism between $H^{2}\left(O_{d}^{+},{ }_{\epsilon} \mathbb{C}_{\epsilon}\right)$ and $o(d, \mathbb{C})$, the space of complex antisymmetic $d \times d$ matrices, and also provides a basis of the former. 


\section{Notations AND PRELIMINARIES}

1.1. Unitary quotient algebras. The object of our study will be augmented algebras $(A, \epsilon)$, i.e. unital $*$-algebras (over $\mathbb{C}$ ) equipped with a unital $*$-homomorphism $\epsilon: A \rightarrow \mathbb{C}$ (a character). We are going to consider only a special kind of augmented algebras. We will assume that $A$ admits a collection of elements $\left\{u_{j k}: j, k=1 \ldots, d\right\}$ (where $d$ is some positive integer) generating $A$ as a *algebra, such that the matrix $u=\left(u_{j k}\right)_{j, k=1}^{d} \in M_{d}(A)$ is unitary (that is, $\left.u u^{*}=u^{*} u=I_{M_{d}(A)}\right)$ and that the character satisfies the formula

$$
\epsilon\left(u_{j k}\right)=\delta_{j k}, j, k=1, \ldots, d .
$$

We shall call such pairs $(A, \epsilon)$ for short unitary quotient algebras, and the reason for that will become clear soon. If the set of generators is fixed, then we will denote a unitary quotient algebra by $(A, u)$, and will occasionally write $d=\operatorname{dim} u$. The character on $(A, u)$ is uniquely determined by the formula (1.1) (note that its existence is assumed in the definition of a unitary quotient algebra).

The key example to keep in mind is the following.

Example 1.1. (Brown-Glockner-von Waldenfels algebra) The Brown-Glockner-von Waldenfels algebra (also called the noncommutative analogue of the algebra of coefficients of the unitary group) $K\langle d\rangle, d \in \mathbb{N}$, is the universal unital *-algebra with $d^{2}$ generators $x_{j k}$ $(j, k=1,2, \ldots, d)$ and the relations making the matrix $x:=\left(x_{j k}\right)_{j, k=1}^{d}$ unitary. Then $(K\langle d\rangle, x)$ is a unitary quotient algebra, and all other unitary quotient algebras are quotients of $(K\langle d\rangle, x)$.

More examples of unitary quotients algebras come from compact matrix quantum groups in the sense of Woronowicz ([Wo 1$]$. More precisely, every compact matrix quantum group $\mathbb{G}=(\mathrm{A}, u)$ with a unital $\mathrm{C}^{*}$-algebra $\mathrm{A}$ and a fundamental representation $u$ of $\mathbb{G}$, gives rise to a structure of a unitary quotient algebra $(A, u)$ with $A$ being the unital *algebra $\operatorname{Pol}(\mathbb{G})$ spanned by coefficients of irreducible representations of $\mathbb{G}$ and the same $u$. The character $\epsilon$ is the counit. Note that the pair $(\operatorname{Pol}(\mathbb{G}), \epsilon)$ is not only an augmented algebra, but also has the structure of a Hopf $*$-algebra (i.e. it admits a comultiplication and an antipode). In the situations where this additional structure plays a role (e.g. Lemma 1.12), it will be convenient to refer to unitary quotient algebras $(A, u)$ arising from compact matrix quantum groups as $C M Q G$ (compact-matrix-quantum-group) algebras.

Contrary to the Brown-Glockner-von Waldenfels algebra, the canonical generators of CMQG algebras will always satisfy certain additional relations required by the fact that also $u^{t}$, the transpose of $u$, needs to be invertible. Here, we use the following notation: for a matrix $a=\left(a_{j k}\right)_{j, k=1}^{d} \in M_{d}(A)$ of elements of a ${ }^{*}$-algebra, its transpose will be $a^{t}:=\left(a_{k j}\right)_{j, k=1}^{d}$ and its entry-wise conjugate $\bar{a}:=\left(a_{j k}^{*}\right)_{j, k=1}^{d}$.

A crucial notion in our approach is that of a quotient. Given two unitary quotient algebra, $(A, u)$ and $(B, v)$, we will say that $(B, v)$ is a quotient of $(A, u)$ if $\operatorname{dim} u=\operatorname{dim} v$ and there is a unital *-homomorphism $q: A \rightarrow B$ such that

$$
q\left(u_{j k}\right)=v_{j k}, \quad j, k=1, \ldots, \operatorname{dim} v .
$$

Note that then $q$ is automatically surjective, and preserves the character (we have $\left.\left(\epsilon_{B} \circ q\right)\left(u_{j k}\right)=\epsilon_{B}\left(v_{j k}\right)=\delta_{j k}=\epsilon_{A}\left(u_{j k}\right)\right)$. As mentioned in Example 1.1. if $(A, u)$ is a unitary quotient algebra with $d=\operatorname{dim} u$, then it is a quotient of $K\langle d\rangle$ (noncommutative analogue of the algebra of coefficients of the unitary group), which explains the terminology. Let us also remark that if $A=\operatorname{Pol}(\mathbb{G})$, and $A=\operatorname{Pol}(\mathbb{H})$, for $\mathbb{G}, \mathbb{H}$ compact matrix quantum 
groups and with $u, v$ being respective fundamental representations of $\mathbb{G}$ and $\mathbb{H}$, the above implies in particular that $\mathbb{H}$ is a quantum subgroup of $\mathbb{G}$ (in the sense introduced in [Pod] and later studied for example in [DKSS]).

The examples of unitary quotient algebras which are of importance for our considerations are the following.

Example 1.2. (Universal unitary quantum group) Consider a matrix $F \in G L_{d}(\mathbb{C})$ $(d \in \mathbb{N})$ and the universal unital *-algebra generated by $d^{2}$ elements $u_{j k}(j, k=1,2, \ldots d)$ such that the matrix $u:=\left(u_{j k}\right)_{j, k=1}^{d}$ is unitary and its conjugate, $\bar{u}$, is similar to a unitary via $F$. More specifically,

$$
\text { (R1) } u u^{*}=I=u^{*} u \text {; }
$$$$
\text { (R2) } F \bar{u} F^{-1}\left(F \bar{u} F^{-1}\right)^{*}=I=\left(F \bar{u} F^{-1}\right)^{*} F \bar{u} F^{-1} \text {. }
$$

The algebra introduced above, denoted by $\operatorname{Pol}\left(U_{F}^{+}\right)$, is a CMQG algebra of the compact quantum group $U_{F}^{+}$, called the universal unitary quantum group (in the sense of Banica, cf. $\left.\mathrm{Ban}_{2}\right)$. Hence the pair $\left(\operatorname{Pol}\left(U_{F}^{+}\right), u\right)$ is a unitary quotient algebra. In the particular case when $F=I_{d}$ we write $U_{d}^{+}:=U_{I_{d}}^{+}$. The compact quantum group $U_{d}^{+}$is called the free unitary quantum group.

The universality of the family $\left\{U_{F}^{+} ; K \in G L_{d}(\mathbb{C})\right\}$ is understood as follows (cf. $\left.\mathrm{Ban}_{2}\right)$. Given a compact quantum matrix group $\mathbb{G}$ with the fundamental representation $w:=\left(w_{j k}\right)_{j, k=1}^{d} \in$ $M_{d}(\operatorname{Pol}(\mathbb{G}))$, we know from $\mathrm{Wo}_{1}$ that the matrix $\bar{w}$ is equivalent to a unitary representation, hence there exists an invertible complex matrix $F \in M_{d}(\mathbb{C})$ such that $F \bar{w} F^{-1}$ is unitary. Then $(\operatorname{Pol}(\mathbb{G}), w)$ is a quotient of $\left(\operatorname{Pol}\left(U_{F}^{+}\right), u\right)$.

Let us observe that the relation (R2) is equivalent to the equality

$$
u^{t} Q \bar{u} Q^{-1}=I=Q \bar{u} Q^{-1} u^{t} .
$$

with the positive matrix $Q=F^{*} F$. Thus $\operatorname{Pol}\left(U_{F}^{+}\right)$is isomorphic to the CMQG algebra associated with the universal unitary quantum group in the sense of Van Daele and Wang, cf. [VDW], defined as the universal unital *-algebra $A_{u}^{W}(Q)$ generated by the coefficients of $u:=\left(u_{j k}\right)_{j, k=1}^{d}$ satisfying (R1) and (R3). In other words, $\operatorname{Pol}\left(U_{F}^{+}\right)$is the quotient of $K\langle d\rangle$ by the ideal generated by the relations ( $(\overline{R 3})$, i.e. $u^{t} Q \bar{u} Q^{-1}=I=Q \bar{u} Q^{-1} u^{t}$ with $Q=F^{*} F$.

Remark 1.3. It follows from Proposition 6.4.7 in [Tim] that up to an isomorphism of CMQG algebras without loss of generality we can assume that the matrix $Q$ is diagonal. In that case, the relation ( $(\underline{\mathrm{R}} 3)$ reads

$$
\sum_{p=1}^{d} \frac{Q_{p p}}{Q_{k k}} u_{p j} u_{p k}^{*}=\delta_{j k} 1, \quad \sum_{p=1}^{d} \frac{Q_{j j}}{Q_{p p}} u_{j p}^{*} u_{k p}=\delta_{j k} 1, \quad j, k=1, \ldots, d .
$$

Example 1.4. (Quantum group $\left.S U_{q}(d)\right)$ Let $d \in \mathbb{N}$ and $q \in(0,1)$. The algebra $\operatorname{Pol}\left(S U_{q}(d)\right.$ ) is defined as the universal unital *-algebra generated by the matrix coefficients of the matrix $u=\left(u_{j k}\right)_{j, k=1}^{d}$ satisfying the unitarity condition (R1) and the quantum determinant condition

$$
\sum_{\sigma \in S_{d}}(-q)^{i(\sigma)} u_{\sigma(1), \tau(1)} u_{\sigma(2), \tau(2)} \ldots u_{\sigma(d), \tau(d)}=(-q)^{i(\tau)} \cdot 1, \quad \tau \in S_{d},
$$

where $S_{d}$ denotes the permutation group on $d$ letters and $i(\tau)$ denotes the number of inversions of the permutation $\tau$ (see $\left[\mathrm{Wo}_{2}\right)$. 
It is known, see [VD, Lemma 4.7], that the adjoint $\bar{u}$ of the representation $u$ of $S U_{q}(d)$, is similar to a unitary via the matrix $F=\left(q^{j-d} \delta_{j k}\right)_{j, k=1}^{d}$. Hence the ${ }^{*}$-algebra $\operatorname{Pol}\left(S U_{q}(d)\right)$ is the quotient of $\operatorname{Pol}\left(U_{F}^{+}\right)$by the ideal generated by the twisted determinant condition (1.4).

Example 1.5. (Universal orthogonal quantum group) Let $d \in \mathbb{N}$ and let $F \in G L_{d}(\mathbb{C})$ satisfy the condition $F \bar{F}= \pm I$. The universal *-algebra generated by $d^{2}$ elements $v_{j k}, j, k=$ $1, \ldots d$ subject to the condition that the matrix $v=\left(v_{j k}\right)$ is unitary and $v=F \bar{v} F^{-1}$, is a CMQG algebra associated to the universal orthogonal compact quantum group, denoted by $O_{F}^{+}\left(\left[\operatorname{Ban}_{1}\right]\right)$. Therefore we denote it by $\operatorname{Pol}\left(O_{F}^{+}\right)$. Again we write $O_{d}^{+}:=O_{I_{d}}^{+}$and call $O_{d}^{+}$the free orthogonal quantum group.

Note that $\operatorname{Pol}\left(O_{F}^{+}\right)$is the quotient of $K\langle d\rangle$ and of $\operatorname{Pol}\left(U_{F}^{+}\right)$by the ideal generated by the relations $u=F \bar{u} F^{-1}$, or equivalently, $u F=F \bar{u}$. In particular, $\operatorname{Pol}\left(O_{d}^{+}\right)$is the quotient of $K\langle d\rangle$ by the ideal generated by the relations $u_{j k}=u_{j k}^{*}, j, k=1, \ldots, d$.

Remark 1.6. Different matrices $F$ can lead to isomorphic quantum groups: the quantum groups $O_{F}^{+}$and $O_{F^{\prime}}^{+}$are isomorphic (so in particular $\operatorname{Pol}\left(O_{F}^{+}\right)$and $\operatorname{Pol}\left(O_{F^{\prime}}^{+}\right)$are isomorphic as *-algebras with characters) if and only if $F^{\prime}=U F U^{t}$ for a unitary matrix $U$ by Tim, Proposition 6.4.7] (see also [VDW]). The matrices $F$ representing each class of isomorphic quantum groups can be classified as follows (see [Ri, Remark 1.5.2]):

(1) either there exists a diagonal matrix $D=\operatorname{diag}\left(\lambda_{1}, \ldots, \lambda_{p}\right)$ with $0<\lambda_{1} \leq \ldots \leq \lambda_{p}<1$ and then

$$
F=\left(\begin{array}{ccc}
0 & D & 0 \\
D^{-1} & 0 & 0 \\
0 & 0 & I_{d-2 p}
\end{array}\right)
$$

(2) or there exists a diagonal matrix $D=\operatorname{diag}\left(\lambda_{1}, \ldots, \lambda_{\frac{d}{2}}\right)$ with $0<\lambda_{1} \leq \ldots \leq \lambda_{\frac{d}{2}} \leq 1$ and then

$$
F=\left(\begin{array}{cc}
0 & D \\
-D^{-1} & 0
\end{array}\right)
$$

Let us finally note that, in the definition of $O_{F}^{+}$, we could have allowed also matrices $F$ such that $F \bar{F}=c I$ for any non-zero $c \in \mathbb{R}$, but this does not increase the class of quantum groups in question.

Example 1.7. (Cocommutative quantum groups) Let $\Gamma$ be a finitely generated group, with the fixed generating set $\gamma_{1}, \ldots, \gamma_{d}$. Then the complex group algebra $\mathbb{C}[\Gamma]$ with the matrix $u \in M_{d}(\mathbb{C}[\Gamma])$ given by the formula $u=\left(\delta_{j k} \gamma_{j}\right)_{j, k=1}^{d}$ is a unitary quotient algebra. In fact, $(\mathbb{C}[\Gamma], u)$ is a cocommutative (sometimes also called abelian) CMQG algebra. One shows that all cocommutative CMQG algebras arise in this way, and we view the corresponding compact quantum group as the dual of $\Gamma$, writing $\mathbb{C}[\Gamma]=\operatorname{Pol}(\hat{\Gamma})$.

1.2. Properties (LK), (NC), (GC) - definitions and known results. Let $(A, \epsilon)$ be a unital *-algebra with a character (for simplicity we assume from the beginning that $A$ is a unitary quotient algebra). We say that a linear map $\psi: A \rightarrow \mathbb{C}$ is a generating functional if it is:

- normalized, i.e. $\psi(1)=0$;

- hermitian, i.e. $\psi\left(a^{*}\right)=\overline{\psi(a)}$ for all $a \in A$;

- conditionally positive, i.e. $\psi\left(a^{*} a\right) \geq 0$ for $a \in \operatorname{ker} \epsilon$. 
The name comes from the correspondence between such functionals and weakly continuous convolution semigroups of states on $A$, for which the generating functionals are infinitesimal generators. Such semigroups are in turn in one-to-one correspondence with Lévy processes on $A$, cf. $\left[\mathrm{Sch}_{2}\right.$, Corollary 1.9.7], [FS, Proposition 4.3.2]. We refer to $\left[\mathrm{Sch}_{2}\right.$ and $\left[\mathrm{Sch}_{1}\right.$ for more information on the related concepts.

Definition 1.8. A Schürmann triple on an augmented algebra $(A, \epsilon)$ is a triple $(\rho, \eta, \psi)$, consisting of a unital *-representation $\rho: A \rightarrow L_{\text {ad }}(D)$ by adjointable linear operators on a pre-Hilbert space $D$, a linear map $\eta: A \rightarrow D$ such that

$$
\eta(a b)=\rho(a) \eta(b)+\eta(a) \epsilon(b), \quad a, b \in A,
$$

and a linear functional $\psi: A \rightarrow \mathbb{C}$ such that

$$
\psi(a b)=\psi(a) \epsilon(b)+\epsilon(a) \psi(b)+\left\langle\eta\left(a^{*}\right), \eta(b)\right\rangle, \quad a, b \in A,
$$

Condition (1.5) means that $\eta$ is a $1-\rho-\epsilon$-cocycle, and Condition (1.6) is equivalent to the fact that the bilinear map $A \times A \ni(a, b) \mapsto\left\langle\eta\left(a^{*}\right), \eta(b)\right\rangle \in \mathbb{C}$ is the 2- $\epsilon$ - $\epsilon$-coboundary of $\psi$. See the beginning of Section 4 for a systematic introduction of the terminology of Hochschild cohomology.

Schürmann showed via a GNS-type construction that for every generating functional such a triple exists, cf. [Sch , Theorem 2.3.4], [FS, Section 4.4].

The unitarity relations imply that the generators $\left\{u_{j k} ; j, k=1, \ldots, d\right\}$ of a unitary quotient algebra $A$ are always represented by bounded operators, and therefore we can extend $\rho(a)$ for all $a \in A$ to a bounded operator on the completion $H=\bar{D}$. For this reason we can view the maps $\rho$ and $\eta$ of a Schürmann triple on a unitary quotient algebra as taking values in $B(H)$ and $H$, resp., with $H$ a Hilbert space.

The triple is unique, up to a suitable notion of a unitary isomorphism, provided $\eta(A)$ is dense in $H$. It is called the Schürmann triple on $A$ associated to $\psi$. Conversely, given any Schürmann triple on $A$ the 'hermitianisation' of its third ingredient (i.e. the functional obtained by passing from $\psi$ to $\frac{1}{2}(\psi+\bar{\psi})$, where $\bar{\psi}(a):=\overline{\psi\left(a^{*}\right)}$ for all $a \in A$ ) is a generating functional. Thus we have a natural one-to-one correspondence between Schürmann triples on $A$ with a hermitian functional and generating functionals on $A$.

In what follows whenever we speak about a representation of a unital *-algebra on a Hilbert space we mean a unital *-representation.

We say that a pair $(\rho, \eta)$ of a representation $\rho$ and a $\rho$ - $\epsilon$-cocycle $\eta$ admits a generating functional if there exists a linear functional $\psi$ such that $(\rho, \eta, \psi)$ is a Schürmann triple. In general, a pair $(\rho, \eta)$, consisting of a representation and a $\rho$ - $\epsilon$-cocycle, need not admit a generating functional. This is basically due to the coboundary condition (1.6) which implies that the values of $\psi$ on $K_{2}=\operatorname{Span}\{a b ; a, b \in \operatorname{ker} \epsilon\}$ have to satisfy $\psi(a b)=\left\langle\eta\left(a^{*}\right), \eta(b)\right\rangle$ for $a, b \in \operatorname{ker} \epsilon$. It is easy to find an augmented algebra $(A, \epsilon)$ with a cocycle $\eta$ and elements $a, b, a^{\prime}, b^{\prime} \in \operatorname{ker} \epsilon$ such that $a b=a^{\prime} b^{\prime}$, but $\left\langle\eta\left(a^{*}\right), \eta(b)\right\rangle \neq\left\langle\eta\left(\left(a^{\prime}\right)^{*}\right), \eta\left(b^{\prime}\right)\right\rangle$, cf. [Ske 3 , Example 2.1] or the proof of Proposition 2.3.

A generating functional $\psi$ is called Gaussian if it vanishes on triple products of elements from the kernel of the counit: $\psi(a b c)=0$ for $a, b, c \in \operatorname{ker} \epsilon$. This is the case if and only if the associated representation $\rho$ in the Schürmann triple $(\rho, \eta, \psi)$ is of the form $\rho=\epsilon(\cdot)$ id or equivalently if the cocycle $\eta$ is Gaussian, i.e. $\eta(a b)=\epsilon(a) \eta(b)+\eta(a) \epsilon(b), a, b \in A$.

Given a representation $\rho$ of $A$ on $H$ we can always extract the maximal Gaussian subspace of the representation space $H$, that is the space $H_{G}:=\{\eta \in H: \rho(a) \eta=\epsilon(a) \eta, a \in A\}$. If 
$H_{G}=\{0\}$, then we say that the representation (and the cocycle, and the associated generating functional) is purely non-Gaussian.

Let $\psi: A \rightarrow \mathbb{C}$ be a generating functional and let $(\rho, \eta, \psi)$ be the associated Schürmann triple with the representation space $H$. Let $P_{G}$ be the projection from $H$ onto the maximal Gaussian subspace $H_{G}$ and set

$$
\rho_{G}=\left.\rho\right|_{H_{G}}, \rho_{N}=\left.\rho\right|_{H_{G}^{\perp}}, \quad \text { and } \quad \eta_{G}=P_{G} \eta, \eta_{N}=\left(I-P_{G}\right) \eta .
$$

Then $\eta_{G}$ is a Gaussian cocycle and $\eta_{N}$ is a purely non-Gaussian cocycle. If there exist generating functionals $\psi_{G}$ and $\psi_{N}$ such that the $\left(\rho_{G}, \eta_{G}, \psi_{G}\right)$ and $\left(\rho_{N}, \eta_{N}, \psi_{N}\right)$ are Schürmann triples, then we say that $\psi$ admits a Lévy-Khintchine decomposition.

If for a given $\psi$ one of the pairs $\left(\rho_{G}, \eta_{G}\right)$ or $\left(\rho_{N}, \eta_{N}\right)$ admits a generating functional, say $\psi_{G}$, then it follows that $\psi$ admits a Lévy-Khintchine decomposition, since $\psi-\psi_{G}$ will be a generating functional for $\left(\rho_{N}, \eta_{N}\right)$. In fact it is easy to see that $\psi$ admits a Lévy-Khintchine decomposition if and only if it can be written as a sum of two generating functionals, one of which is Gaussian, and the other purely non-Gaussian. This motivated our terminology which comes from the analogy to the Lévy-Khintchine formula in the classical case, where a the characteristic function of a Lévy process is commonly written as the exponential of a sum of a Gaussian term and a purely non-Gaussian term.

Adopting the notation from [FGT], we shall study the following three properties in this paper.

Definition 1.9. We shall say that the augmented algebra $(A, \epsilon)$

- has the property $(\mathrm{LK})$ if any generating functional on $(A, \epsilon)$ admits a Lévy-Khintchine decomposition;

- has the property (GC) if any Gaussian cocycle $\eta: A \rightarrow H$ can be completed to a Schürmann triple $(\epsilon(\cdot)$ id, $\eta, \psi)$;

- has the property (NC) if any pair $(\rho, \eta)$ consisting of a representation $\rho: A \rightarrow B(H)$ and a $\rho$ - $\epsilon$-cocycle $\eta: A \rightarrow H$ with $H_{G}=\{0\}$ can be completed to a Schürmann triple $(\rho, \eta, \psi)$.

Property (LK) is equivalent to Schürmann's property (C), property (GC) was called (C') by Schürmann, and property (NC) was not explicitly named by Schürmann, cf. [Sch 2 , 5.1 Maximal quadratic components].

If $A=\operatorname{Pol}(\mathbb{G})$ is a CMQG algebra, we will also say simply that the compact quantum group $\mathbb{G}$ has the property (LK), (GC), or (NC).

As observed by Schürmann, either (GC) or (NC) implies (LK). However, no two of the conditions are equivalent, as shown by the examples of group rings provided in [FGT]. There exists a cocommutative CMQG-algebra (i.e. a CMQG-algebra coming from a group ring of a finitely generated group, see Example 1.7) without the property (LK). On the other hand, any commutative CMQG-algebra (in other words, any classical compact matrix group) has (LK), see [Sch 1 , Theorem 3.12 (at the top of p. 360)].

For our considerations the key fact will be the following theorem of Schürmann, implying in particular that the algebra $K\langle d\rangle$ has all the properties listed in Definition 1.9.

Theorem 1.10. $\mathrm{Sch}_{1}$, Theorem 3.12(i)] Let $d \in \mathbb{N}$ and $\rho: K\langle d\rangle \rightarrow B(H)$ be a representation of the Brown-Glockner-von Waldenfels algebra on a Hilbert space $H$. Any $\rho$ - $\epsilon$-cocycle $\eta$ on $K\langle d\rangle$ admits a generating functional $\psi: K\langle d\rangle \rightarrow \mathbb{C}$ which is a coboundary for $\eta$. A possible choice of the functional $\psi$ is determined by the following formula describing its value on the 
canonical generators of $K\langle d\rangle(j, k=1, \ldots, d)$ :

$$
\psi\left(x_{j k}\right)=-\frac{1}{2} \sum_{p=1}^{d}\left\langle\eta\left(x_{j p}^{*}\right), \eta\left(x_{k p}^{*}\right)\right\rangle .
$$

The values of $\psi$ on general elements of $A$ can then be deduced from the normalization $\psi(1)=0$, hermitianity, and the coboundary condition (1.6).

In general we have the freedom to modify the generating functional by any selfadjoint matrix $H \in M_{d}(\mathbb{C})$, by setting

$$
\psi_{H}\left(x_{j k}\right)=-\frac{1}{2} \sum_{p=1}^{d}\left\langle\eta\left(x_{j p}^{*}\right), \eta\left(x_{k p}^{*}\right)\right\rangle+i H_{j k}
$$

for $j, k=1, \ldots, d$.

1.3. Schürmann triples on unitary quotient algebras. In this paper, an important strategy for associating a generating functional to a given cocycle on a unitary quotient algebra $(A, u)$ relies on the fact that $A$ is a quotient of $K\langle d\rangle$, arising via imposing on the generators $u_{j k}$ some additional relations. We will explain it now.

Let $(A, u)$ be a unitary quotient algebra. Note first that since $A$ arises as the universal *-algebra generated by the coefficients $\left(u_{j k}: j, k=1, \ldots, d\right)$ satisfying certain relations, then any representation $\rho$ of $B$, any $\rho$ - $\epsilon$-cocycle $\eta$ and a generating functional $\psi$ for $\eta$ (if it exists) are all determined by the values they take on the canonical generators and their adjoints (the values on 1 are fixed: $\rho(1)=I, \eta(1)=0$ and $\psi(1)=0)$.

Observe that a given cocycle $\eta$ on $A$ lifts to a cocycle $\eta^{\prime}$ on $K\langle d\rangle$ simply by composition with the quotient map. We can therefore use Theorem 1.10 and Schürmann's formula (1.7) to define a generating functional $\psi^{\prime}$ on $K\langle d\rangle$ associated to the cocycle $\eta^{\prime}$. This generating functional will then descend to $A$ if and only if it preserves the extra relations in $A$, i.e., it vanishes on the kernel of the canonical quotient map from $K\langle d\rangle$ to $A$. To show the existence of a generating functional $\psi$ on $A$ it suffices to show that the values on the generators, proposed by (1.7), are compatible with the relations determining $A$. This result was proven in [FKS] we recall it here as we will use it many times in this paper.

Lemma 1.11. [FKS, Lemma 5.8] Let $B$ be $a^{*}$-algebra generated by a collection of elements, $a_{1}, \ldots, a_{n}$, let $\epsilon$ be a character on $B$, and let $(\rho, \eta, \psi)$ be a Schürmann triple on $B$. Let $A$ be the quotient of $B$ by the two-sided ideal generated by the selfadjoint relations $r_{1}\left(a_{1}, \ldots, a_{n}\right)=0$, $\ldots, r_{k}\left(a_{1}, \ldots, a_{n}\right)=0$.

If the maps $\epsilon, \rho, \eta$, and $\psi$ vanish on $r_{1}, \ldots, r_{k}$, then $(\rho, \eta, \psi)$ is a Schürmann triple on $A$.

We finish this section by providing a general result concerning an automatic property of Gaussian cocycles on CMQG algebras.

Lemma 1.12. Suppose that $(A, u)$ is a CMQG algebra, $H$ is a Hilbert space and $\eta: A \rightarrow H$ is a Gaussian cocycle. Then

$$
\eta\left(u_{j k}^{*}\right)=-\eta\left(u_{k j}\right), \quad j, k=1, \ldots, d .
$$

Proof. Note that the CMQG algebra $(A, u)$ has a Hopf $*$-algebra structure with comultiplication $\Delta$ and an antipode $S$ which acts on the generators as $S\left(u_{j k}\right)=u_{k j}^{*}$. 
Let $a \in A$, and use the Sweedler notation $\Delta(a)=a_{(1)} \otimes a_{(2)} \in A \otimes A$. Applying $\eta$ to the formula $\epsilon(a) 1=a_{(1)} S\left(a_{(2)}\right)$ yields

$$
0=\eta(\epsilon(a) 1)=\eta\left(a_{(1)}\right) \epsilon\left(a_{(2)}\right)+\eta\left(S a_{(1)}\right) \epsilon\left(a_{(2)}\right)=\eta(a)+\eta(S a) .
$$

This shows that $\eta=-\eta \circ S$, from which the statement follows.

\section{Generic CASE FOr $U_{F}^{+}$AND $O_{F}^{+}$}

Throughout this section we consider the quantum groups $U_{F}^{+}$and $O_{F}^{+}$with a matrix $F \in$ $G L_{d}(\mathbb{C})$ such that $Q=F^{*} F \in G L_{d}(\mathbb{C})$ has pairwise distinct eigenvalues. We will call such $F$ generic. Note that genericity is preserved under the transformations $F \mapsto w^{*} F w$ and $F \mapsto w F w^{t}$, if $w \in U_{d}(\mathbb{C})$.

Theorem 2.1. Let $d \in \mathbb{N}$ and let $F \in G L_{d}(\mathbb{C})$ be a generic matrix. Then $U_{d}^{+}(F)$ has the property (GC), hence also the property (LK).

Proof. According to Remark 1.3, we may and do assume that the matrix $Q=F^{*} F$ is diagonal and use the presentation of $\operatorname{Pol}\left(U_{F}^{+}\right)$as $A:=A_{u}^{W}(Q)$. Suppose that $H$ is a Hilbert space and $\eta: \operatorname{Pol}\left(U_{F}^{+}\right) \rightarrow H$ is a Gaussian cocycle. Applying $\eta$ to both sides of the first equality in (1.3) yields $(j, k=1, \ldots, d)$

$$
0=\frac{1}{Q_{k k}} \sum_{p=1}^{d} Q_{p p}\left[\eta\left(u_{p j}\right) \delta_{p k}+\eta\left(u_{p k}^{*}\right) \delta_{p j}\right]=Q_{k k} \eta\left(u_{k j}\right)+Q_{j j} \eta\left(u_{j k}^{*}\right) .
$$

Using Lemma 1.12 we obtain

$$
\left(Q_{k k}-Q_{j j}\right) \eta\left(u_{j k}\right)=0 .
$$

By genericity assumption $Q_{j j} \neq Q_{k k}$ for $j \neq k$. Thus we must have

$$
\eta\left(u_{j k}\right)=0, \quad j, k=1, \ldots, d, \quad j \neq k .
$$

Let $q: K\langle d\rangle \longrightarrow \operatorname{Pol}\left(U_{F}^{+}\right)$be the canonical quotient map, that is the surjective ${ }^{*}$-homomorphism given by $q\left(x_{j k}\right)=u_{j k}, j, k=1, \ldots, d$, where $\left\{x_{j k}: j, k=\ldots, d\right\}$ are generators of $K\langle d\rangle$. The kernel of $q$ is the two-sided ideal generated by the relations

$$
\left.r_{j k}^{(1)}:=\frac{1}{Q_{k k}} \sum_{p=1}^{d} Q_{p p} x_{p j} x_{p k}^{*}-\delta_{j k} 1=0 \quad \text { and } \quad r_{j k}^{(2)}:=Q_{j j} \sum_{p=1}^{d} \frac{1}{Q_{p p}} x_{j p}^{*} x_{k p}-\delta_{j k} 1\right)=0,
$$

$j, k=1, \ldots, d$.

Define $\eta^{\prime}:=\eta \circ q: K\langle d\rangle \longrightarrow H$. It is easy to check that $\eta^{\prime}$ is a Gaussian cocycle on $K\langle d\rangle$. By Theorem 1.10, we can associate with $\eta^{\prime}$ a generating functional $\psi^{\prime}: K\langle d\rangle \longrightarrow \mathbb{C}$, determined by formula (1.7). We would like to define a functional $\psi: A \longrightarrow \mathbb{C}$ such that $\psi(q(a)):=\psi^{\prime}(a)$. According to Lemma 1.11, to prove that $\psi$ is well-defined, it is enough to

check that $\psi^{\prime}\left(r_{j k}^{(m)}\right)=0$ for $j, k=1, \ldots, d, m=1,2$. We only show the case $m=1$ as the proof of the second case is very similar.

By the first part of the proof $\eta$ (so also $\eta^{\prime}$ ) vanishes on the off-diagonal generators, therefore the definition (1.7) of $\psi^{\prime}$ finally gets the form

$$
\psi^{\prime}\left(x_{j k}\right)=0 \quad(j \neq k) \quad \text { and } \quad \psi^{\prime}\left(x_{k k}\right)=-\frac{1}{2}\left\|\eta^{\prime}\left(x_{k k}\right)\right\|^{2} .
$$


For $j, k=1, \ldots, d$ and $j \neq k$ we have

$$
\begin{aligned}
\psi^{\prime}\left(\sum_{p=1}^{d} Q_{p p} x_{p j} x_{p k}^{*}\right) & =\sum_{p=1}^{d} Q_{p p} \psi^{\prime}\left(x_{p j} x_{p k}^{*}\right) \\
& =\sum_{p=1}^{d} Q_{p p}\left[\psi^{\prime}\left(x_{p j}\right) \delta_{p k}+\delta_{p j} \psi^{\prime}\left(x_{p k}^{*}\right)+\left\langle\eta^{\prime}\left(x_{p j}^{*}\right), \eta^{\prime}\left(x_{p k}^{*}\right)\right\rangle\right] \\
& =Q_{k k} \psi^{\prime}\left(x_{k j}\right)+Q_{j j} \psi^{\prime}\left(x_{j k}^{*}\right)+\sum_{p=1}^{d} Q_{p p}\left\langle\eta^{\prime}\left(x_{p j}^{*}\right), \eta^{\prime}\left(x_{p k}^{*}\right)\right\rangle .
\end{aligned}
$$

The first two summands vanish due to the definition of $\psi^{\prime}$, see (2.3). Moreover by Lemma 1.12 $\eta^{\prime}\left(x_{j k}^{*}\right)=\eta\left(u_{j k}^{*}\right)=-\eta\left(u_{k j}\right)=0$ for $j \neq k$, and hence the last summand must be proportional to $\delta_{p, j} \delta_{p, k}$. Therefore, (2.4) is zero for $j \neq k$.

On the other hand if $j=k$, then again by Lemma 1.12 we get

$$
\psi^{\prime}\left(\sum_{p=1}^{d} Q_{p p} x_{p j} x_{p j}^{*}\right)=Q_{j j}\left[\psi^{\prime}\left(x_{j j}\right)+\psi^{\prime}\left(x_{j j}^{*}\right)+\left\|\eta^{\prime}\left(x_{j j}\right)\right\|^{2}\right] .
$$

Using the fact that $\psi^{\prime}\left(x_{k k}\right)=\psi^{\prime}\left(x_{k k}^{*}\right)=-\frac{1}{2}\left\|\eta^{\prime}\left(x_{k k}\right)\right\|^{2}$ for all $k=1,2, \ldots, d$, we see that the above expression also vanishes. Thus the prescription $\psi(q(a)):=\psi^{\prime}(a)$ for all $a \in A$ is well-defined. Now a routine check shows that the coboundary of this functional is the bilinear form $(a, b) \mapsto\left\langle\eta\left(a^{*}\right), \eta(b)\right\rangle$.

We shall see later (see Theorem 3.5) that genericity of $F$ is not only sufficient, but also a necessary condition for the quantum group $U_{F}^{+}$to have property $(\mathrm{GC})$.

The analogue of Theorem 2.1 holds also in the orthogonal case, as we show in the next theorem.

Theorem 2.2. Let $d \in \mathbb{N}$ and let $F \in G L(d)$ be a generic matrix such that $F \bar{F}= \pm I$. Then the quantum group $O_{F}^{+}$has the property $(G C)$, hence also the property (LK).

Proof. Let $q: \operatorname{Pol}\left(U_{F}^{+}\right) \rightarrow \operatorname{Pol}\left(O_{F}^{+}\right)$be the canonical quotient map, i.e. the surjective *homomorphism such that $q\left(u_{j k}\right)=v_{j k}, j, k=1 \ldots d$. The kernel of $q$ is generated by the relation $u F=F \bar{u}$, that is by the family of relations

$$
\sum_{p=1}^{d} u_{j p} F_{p k}=\sum_{p=1}^{d} F_{j p} u_{p k}^{*}, \quad j, k=1 \ldots d .
$$

Note first that, without loss of generality, we can assume that $F$ is of the form described in Remark 1.6. Then for each $j=1, \ldots, d$ there exists exactly one $\hat{j} \in\{1, \ldots, d\}$ such that $F_{j \hat{j}} \neq 0$, and similarly, for each $k=1, \ldots, d$ there exists exactly one $\check{k} \in\{1, \ldots, d\}$ such that $F_{\breve{k} k} \neq 0$. The mapings $j \mapsto \hat{j}$ and $k \mapsto \check{k}$ are inverses of one another. All this implies that the kernel of the quotient map $q$ is generated by the relation

$$
u_{j \breve{k}} F_{\breve{k} k}=F_{j \hat{j}} u_{j k}^{*}, \quad j, k=1, \ldots, d .
$$

Let us fix a Hilbert space $H$ and a Gaussian cocycle $\eta: \operatorname{Pol}\left(O_{F}^{+}\right) \longrightarrow H$. We will show that it admits a generating functional. Note first that (2.5) together with Lemma 1.12 imply 
that, for $j, k=1, \ldots, d$,

$$
\eta\left(u_{j \check{k}}\right) F_{\breve{k} k}=-F_{j \hat{j}} \eta\left(u_{k \hat{j}}\right) .
$$

In particular $\eta\left(u_{\breve{k} \check{k}}\right) F_{\breve{k} k}=-F_{\breve{k} k} \eta\left(u_{k k}\right)$, so that

$$
\eta\left(u_{k k}\right)=-\eta\left(u_{\check{k} \check{k}}\right) .
$$

Define now $\eta_{u}:=\eta \circ q: U_{F}^{+} \longrightarrow H$. This is a Gaussian cocycle on $U_{F}^{+}$, so by Theorem 2.1 and its proof, it admits a generating functional $\psi_{u}$ defined on the generators by the formulas $(j, k=1, \ldots, d)$

$$
\psi_{u}\left(u_{j k}\right)= \begin{cases}0 & \text { for } j \neq k, \\ -\frac{1}{2}\left\|\eta_{u}\left(u_{k k}\right)\right\|^{2} & \text { for } j=k .\end{cases}
$$

As before, we would like to define $\psi$ on $O_{F}^{+}$by $\psi(q(a))=\psi_{u}(a), a \in O_{F}^{+}$. Such a functional - if well-defined - will indeed be a generating functional that we seek. It thus remains to check that $\psi_{u}$ vanishes on $\operatorname{ker} q$, or by Lemma 1.11 that we have

$$
\psi_{u}\left(u_{j \breve{k}} F_{\breve{k} k}\right)=\psi_{u}\left(F_{j \hat{j}} u_{\hat{j} k}^{*}\right), \quad j, k=1, \ldots, d .
$$

Note then that for $j, k$ as above

$$
\begin{aligned}
& \psi_{u}\left((u F)_{j k}\right)=F_{\check{k} k} \psi_{u}\left(u_{j \check{k}}\right)=\left\{\begin{array}{ll}
-\frac{F_{\breve{k} k}}{2}\left\|\eta_{u}\left(u_{\breve{k} \check{k}}\right)\right\|^{2} & \text { if } j=\check{k} \\
0 & \text { if } j \neq \check{k}
\end{array},\right. \\
& \psi_{u}\left((F \bar{u})_{j k}\right)=F_{j \hat{j}} \overline{\psi_{u}\left(u_{\hat{j} k}\right)}=\left\{\begin{array}{ll}
-\frac{F_{j \hat{j}}}{2}\left\|\eta_{u}\left(u_{\hat{j} \hat{j}}\right)\right\|^{2} & \text { if } \hat{j}=k \\
0 & \text { if } j_{0} \neq k
\end{array} .\right.
\end{aligned}
$$

Now observe that $j=\check{k}$ if and only if $\hat{j}=k$. Thus the formulas above and the equality (2.6) guarantee that (2.7) always holds.

Note that Theorem 2.2 provides an alternative proof of the property (GC) for $S U_{q}(2)$, $q \in(0,1)$, originally established by Schürmann and Skeide in $[\mathrm{ScS}]$. Indeed, $S U_{q}(2) \cong O_{F}^{+}$ with the generic $F=\left(\begin{array}{cc}0 & 1 \\ -q^{-1} & 0\end{array}\right)$, see [Tim, Proposition 6.4.8], or with $F=\left(\begin{array}{cc}0 & 1 \\ -q^{-1} & 0\end{array}\right)$ (see Example 1.4).

We will now show that the case $S U_{q}(d)$ with $d \geq 3$ is different from that of $d=2$.

Proposition 2.3. Let $d \geq 3$ and $q \in(0,1)$. The quantum group $S U_{q}(d)$ does not have $(G C)$.

Proof. We know from Example 1.4 that $\operatorname{Pol}\left(S U_{q}(d)\right)$ is a quotient of $\operatorname{Pol}\left(U_{F}^{+}\right)$by the ideal generated by the twisted determinant condition (1.4) with a generic matrix $F=\left(q^{j-d} \delta_{j k}\right)_{j, k=1}^{d}$.

Consider first the case of $d=3$ and let $u=\left(u_{j k}\right)_{j, k=1}^{3}$. Set

$$
\eta\left(u_{11}\right):=-1-i, \eta\left(u_{22}\right):=1, \eta\left(u_{33}\right):=i, \eta\left(u_{j k}\right)=0 \text { for } j \neq k
$$

(with $i$ being the complex unit) and $\eta\left(u_{j k}^{*}\right):=-\eta\left(u_{k j}\right)$ for all $j, k=1 \ldots, 3$, and extend it to the whole algebra by the formula $\eta(a b)=\epsilon(a) \eta(b)+\eta(a) \epsilon(b)\left(a, b \in \operatorname{Pol}\left(S U_{q}(3)\right)\right)$. We can check that such $\eta$ vanishes on the relations (R1) and (1.4) defining $S U_{q}(3)$, hence by Lemma 1.11 it defines a Gaussian cocycle on $\operatorname{Pol}\left(S U_{q}(3)\right)$ with values in $\mathbb{C}$. We will write simply $\eta_{j}=\eta\left(u_{j j}\right)$ for $1 \leq j \leq 3$. 
Suppose now that $\eta$ admits a generating functional $\psi: \operatorname{Pol}\left(S U_{q}(3)\right) \rightarrow \mathbb{C}$. Then, computing the value of $\psi$ on both sides of (1.4), and writing for simplicity $\left(t_{1}, t_{2}, t_{3}\right)$ for $(\tau(1), \tau(2), \tau(3))$ and $\left(s_{1}, s_{2}, s_{3}\right)$ for $(\sigma(1), \sigma(2), \sigma(3))$, for any $\tau \in S_{3}$ we would have

$$
\begin{aligned}
0= & \psi\left(\sum_{\sigma \in S_{3}}(-q)^{i(\sigma)} u_{s_{1}, t_{1}} u_{s_{2}, t_{2}} u_{s_{3}, t_{3}}\right) \\
= & \sum_{\sigma \in S_{3}}(-q)^{i(\sigma)}\left[\psi\left(u_{s_{1}, t_{1}}\right) \epsilon\left(u_{s_{2}, t_{2}} u_{s_{3}, t_{3}}\right)+\epsilon\left(u_{s_{1}, t_{1}}\right) \psi\left(u_{s_{2}, t_{2}} u_{s_{3}, t_{3}}\right)+\left\langle\eta\left(u_{s_{1}, t_{1}}^{*}\right), \eta\left(u_{s_{2}, t_{2}} u_{s_{3}, t_{3}}\right)\right\rangle\right] \\
= & \left.(-q)^{i(\tau)} \psi\left(u_{t_{1}, t_{1}}\right)+\sum_{\sigma \in S_{N}}(-q)^{i(\sigma)} \delta_{s_{1}, t_{1}}\left[\psi\left(u_{s_{2}, t_{2}}\right) \delta_{s_{3}, t_{3}}\right)+\delta_{s_{2}, t_{2}} \psi\left(u_{s_{3}, t_{3}}\right)\right] \\
& +\sum_{\sigma \in S_{N}}(-q)^{i(\sigma)} \delta_{s_{1}, t_{1}}\left\langle\eta\left(u_{s_{2}, t_{2}}^{*}\right), \eta\left(u_{s_{3}, t_{3}}\right)\right\rangle+\left\langle\eta\left(u_{s_{1}, t_{1}}^{*}\right), \epsilon\left(u_{s_{2}, t_{2}}\right) \eta\left(u_{s_{3}, t_{3}}\right)+\eta\left(u_{s_{2}, t_{2}}\right) \epsilon\left(u_{s_{3}, t_{3}}\right)\right\rangle \\
= & (-q)^{i(\tau)}\left[\psi\left(u_{t_{1}, t_{1}}\right)+\psi\left(u_{t_{2}, t_{2}}\right)+\psi\left(u_{t_{3}, t_{3}}\right)-\left\langle\eta_{t_{2}}, \eta_{t_{3}}\right\rangle-\left\langle\eta_{t_{1}}, \eta_{t_{3}}\right\rangle-\left\langle\eta_{t_{1}}, \eta_{t_{2}}\right\rangle\right] .
\end{aligned}
$$

We conclude that if $\eta$ admitted a generating functional, then the value

$$
\begin{aligned}
C_{\tau}: & =\left\langle\eta_{t_{1}}, \eta_{t_{2}}\right\rangle+\left\langle\eta_{t_{2}}, \eta_{t_{3}}\right\rangle+\left\langle\eta_{t_{1}}, \eta_{t_{3}}\right\rangle=\left\langle-\eta_{t_{2}}-\eta_{t_{3}}, \eta_{t_{2}}\right\rangle+\left\langle\eta_{t_{2}}, \eta_{t_{3}}\right\rangle+\left\langle-\eta_{t_{2}}-\eta_{t_{3}}, \eta_{t_{3}}\right\rangle \\
& =-\left\|\eta_{t_{2}}\right\|^{2}-\left\|\eta_{t_{3}}\right\|^{2}-\left\langle\eta_{t_{3}}, \eta_{t_{2}}\right\rangle
\end{aligned}
$$

would be independent on $\tau \in S_{3}$. But that is clearly not possible if we consider $\tau=\mathrm{id}$ and $\tau=(23)$, as $\left\langle\eta_{2}, \eta_{3}\right\rangle=i \notin \mathbb{R}$. This proves that $S U_{q}(3)$ does not have (GC).

Embedding the above example into $S U_{q}(d)$ for $d>3$ shows that the latter does not have (GC) either.

Note that, despite ongoing attempts to answer this question, it is still unknown whether $S U_{q}(d)$ (for $d \geq 3$ ) possesses properties $(\mathrm{LK})$ or $(\mathrm{NC})$.

\section{NON-GENERIC CASES}

Throughout this section we will consider quantum groups $U_{F}^{+}$and $O_{F}^{+}$for non-generic matrices $F$. In fact we will mainly focus on the case of $F=I$, as it exhibits the typical non-generic behaviour and can be used to determine other cases.

Given a compact quantum group $\mathbb{G}$ with $\mathbb{G}=U_{d}^{+}$or $\mathbb{G}=O_{d}^{+}$we will denote the canonical fundamental representation by $u=\left(u_{j k}\right)_{j, k=1}^{d} \in M_{d}(\operatorname{Pol}(\mathbb{G}))$. Further given a Hilbert space $H$, a representation $\rho: \operatorname{Pol}(\mathbb{G}) \rightarrow B(H)$, and a $\rho$ - $\epsilon$-cocycle $\eta: \operatorname{Pol}(\mathbb{G}) \rightarrow H$ we will consider the matrices

$$
R=\left(\rho\left(u_{j k}\right)\right)_{j, k=1}^{d} \in M_{d}(B(H)), \quad V=\left(\eta\left(u_{j k}\right)\right)_{j, k=1}^{d} \in M_{d}(H),
$$

and

$$
W=\left(\eta\left(u_{j k}^{*}\right)\right)_{j, k=1}^{d} \in M_{d}(H) .
$$

Finally, given a cocycle $\eta$ as above, it will also be useful to consider the scalar, selfadjoint, matrices

$$
B=\left(\sum_{p=1}^{d}\left\langle\eta\left(u_{j p}\right), \eta\left(u_{k p}\right)\right\rangle\right)_{j, k=1}^{d} \in M_{d}(\mathbb{C})
$$


and

$$
\widetilde{B}=\left(\sum_{p=1}^{d}\left\langle\eta\left(u_{j p}^{*}\right), \eta\left(u_{k p}^{*}\right) \cdot\right\rangle\right)_{j, k=1}^{d} \in M_{d}(\mathbb{C}) .
$$

Note that by Lemma 1.12 for Gaussian cocycles we have $W=-V^{t}$.

We will need later a simple lemma regarding an alternative description of the matrix $B$.

Lemma 3.1. Let $d \in \mathbb{N}$, let $H$ be a Hilbert space, let $\rho: \operatorname{Pol}\left(U_{d}^{+}\right) \rightarrow B(H)$ be a representation, let $\eta: \operatorname{Pol}(\mathbb{G}) \rightarrow H$ be a $\rho$ - $\epsilon$-cocycle and let $B$ be defined as in (3.3). Then we have for all $j, k=1, \ldots, d$

$$
\begin{gathered}
\eta\left(u_{j k}^{*}\right)=-\sum_{p=1}^{d} \rho\left(u_{j p}^{*}\right) \eta\left(u_{k p}\right), \\
B_{j k}=\sum_{p=1}^{d}\left\langle\eta\left(u_{p j}^{*}\right), \eta\left(u_{p k}^{*}\right)\right\rangle .
\end{gathered}
$$

Proof. Using the defining relation $\bar{u} u^{t}=I$, we see that for all $j, k=1, \ldots, d$

$$
0=\eta\left(\sum_{p=1}^{d} u_{j p}^{*} u_{k p}\right)=\sum_{p=1}^{d} \rho\left(u_{j p}^{*}\right) \eta\left(u_{k p}\right)+\eta\left(u_{j k}^{*}\right),
$$

and the first formula in the lemma is proved.

Then

$$
\begin{aligned}
\sum_{p=1}^{d}\left\langle\eta\left(u_{p j}^{*}\right), \eta\left(u_{p k}^{*}\right)\right\rangle & =\sum_{p=1}^{d}\left\langle\sum_{m=1}^{d} \rho\left(u_{p m}^{*}\right) \eta\left(u_{j m}\right), \sum_{m^{\prime}=1}^{d} \rho\left(u_{p m^{\prime}}^{*}\right) \eta\left(u_{k m^{\prime}}\right)\right\rangle \\
& =\sum_{p, m, m^{\prime}=1}^{d}\left\langle\rho\left(u_{p m^{\prime}} u_{p m}^{*}\right) \eta\left(u_{j m}\right), \eta\left(u_{k m^{\prime}}\right)\right\rangle \stackrel{u^{t} \underline{u}=I}{=} \sum_{m=1}^{d}\left\langle\eta\left(u_{j m}\right), \eta\left(u_{k m}\right)\right\rangle=B_{j k} .
\end{aligned}
$$

and the proof is complete.

Proposition 3.2. Let $H$ be a Hilbert space, let $\rho: \operatorname{Pol}\left(U_{d}^{+}\right) \rightarrow B(H)$ be a representation, define the matrix $R$ as in the first formula in (3.1) and let $V \in M_{d}(H)$. Then the following conditions are equivalent:

(i) there is a $\rho$ - $\epsilon$-cocycle $\eta$ on $\operatorname{Pol}\left(U_{d}^{+}\right)$satisfying the second formula in (3.1);

$$
\left(R^{*} V\right)^{t}=\bar{R} V^{t}
$$

Moreover when the above hold, we have $W=-\bar{R} V^{t}$, with $W$ as in (3.2). 
Proof. Suppose first that $\eta$ is a $\rho$ - $\epsilon$-cocycle on $\operatorname{Pol}\left(U_{d}^{+}\right)$and define $R, V$ and $W$ via (3.1) and (3.2). It follows from $u^{*} u=1$ that for each $j, k=1, \ldots, d$

$$
\begin{aligned}
0 & =\eta\left(\delta_{j k} \mathbf{1}\right)=\eta\left(\sum_{p=1}^{d} u_{p j}^{*} u_{p k}\right)=\sum_{p=1}^{d} \rho\left(u_{p j}^{*}\right) \eta\left(u_{p k}\right)+\eta\left(u_{p j}^{*}\right) \epsilon\left(u_{p k}\right) \\
& =\sum_{p=1}^{d}\left(R^{*}\right)_{j p} V_{p k}+W_{j k}=\left(R^{*} V+W^{t}\right)_{j k}
\end{aligned}
$$

and $\bar{u} u^{t}=1$ yields

$$
\begin{aligned}
0 & =\eta\left(\delta_{j k} \mathbf{1}\right)=\eta\left(\sum_{p=1}^{d} u_{j p}^{*} u_{k p}\right)=\sum_{p=1}^{d} \rho\left(u_{j p}^{*}\right) \eta\left(u_{k p}\right)+\eta\left(u_{j p}^{*}\right) \epsilon\left(u_{k p}\right) \\
& =\sum_{p=1}^{d} \bar{R}_{j p} V_{k p}+W_{j k}=\left(\bar{R} V^{t}+W\right)_{j k} .
\end{aligned}
$$

Comparing the two formulea we see that

$$
\left(R^{*} V\right)^{t}=-W=\bar{R} V^{t}
$$

i.e. (3.5) holds.

On the other hand, if we are given a matrix $V \in M_{d}(H)$ which satisfies (3.5), then the mapping defined on the generators $(j, k=1, \ldots, d)$ as $\eta\left(u_{j k}\right)=V_{j k}$ and $\eta\left(u_{j k}^{*}\right)=W_{j k}$, with $W:=-\bar{R} V^{t}$, extends by linearity and the cocycle property to a mapping (a $\rho$ - $\epsilon$-cocycle) on $\operatorname{Pol}\left(U_{d}^{+}\right)$. Indeed, as the calculations in the previous step show, $\eta$ defined this way respects the relations $u^{*} u-1$ and $\bar{u} u^{t}-1$. So we are left to prove that $\eta$ respects the two remaining sets of relations.

Note then that if the equality (3.5) holds and $W:=-\bar{R} V^{t}$, then $-V=\left(R^{t} W\right)^{t}=R W^{t}$. Indeed, as $R^{t} \bar{R}=R R^{*}=I$, we have

$$
\begin{gathered}
\left(R^{t} W\right)^{t}=-\left(R^{t} \bar{R} V^{t}\right)^{t}=-V, \\
R W^{t}=-R\left(\bar{R} V^{t}\right)^{t} \stackrel{(3.5)}{=}-R\left(\left(R^{*} V\right)^{t}\right)^{t}=-R R^{*} V=-V .
\end{gathered}
$$

Thus for any $j, k=1, \ldots, d$

$$
\begin{aligned}
& \eta\left(\left(u u^{*}\right)_{j k}\right)=\sum_{p=1}^{d} \eta\left(u_{j p} u_{k p}^{*}\right)=\left(R W^{t}+V\right)_{j k}=0=\eta\left(\delta_{j k} \mathbf{1}\right) \quad \text { and } \\
& \eta\left(\left(u^{t} \bar{u}\right)_{j k}\right)=\sum_{p=1}^{d} \eta\left(u_{p j} u_{p k}^{*}\right)=\left(R^{t} W+V^{t}\right)_{j k}=0=\eta\left(\delta_{j k} \mathbf{1}\right),
\end{aligned}
$$

so $\eta$ respects also the missing relations.

We are now ready to formulate a criterion for a given cocycle on $\operatorname{Pol}\left(U_{d}^{+}\right)$to admit a generating functional.

Theorem 3.3. Let $H$ be a Hilbert space, let $\rho: \operatorname{Pol}\left(U_{d}^{+}\right) \rightarrow B(H)$ be a representation, and let $\eta: \operatorname{Pol}\left(U_{d}^{+}\right) \rightarrow H$ be a $\rho$ - $\epsilon$-cocycle. Then $\eta$ admits a generating functional if and only if

$$
\widetilde{B}=B^{t}
$$


where $B$ and $\widetilde{B}$ are defined as in (3.3) and (3.4).

Proof. Suppose that $\psi$ is a generating functional associated with $\eta$. Then, due to the relations $u u^{*}=I$ and $\bar{u} u^{t}=I$, we have for each $j, k=1, \ldots, d$

$$
\begin{aligned}
& 0=\delta_{j k} \psi(\mathbf{1})=\sum_{p=1}^{d} \psi\left(u_{j p} u_{k p}^{*}\right)=\psi\left(u_{j k}\right)+\overline{\psi\left(u_{k j}\right)}+\widetilde{B}_{j k}, \\
& 0=\delta_{k j} \psi(\mathbf{1})=\sum_{p=1}^{d} \psi\left(u_{k p}^{*} u_{j p}\right)=\overline{\psi\left(u_{k j}\right)}+\psi\left(u_{j k}\right)+B_{k j} .
\end{aligned}
$$

Comparing the above two equations, we arrive at $\widetilde{B}=B^{t}$, as required.

Conversely suppose that the cocycle $\eta$ satisfies $\widetilde{B}=B^{t}$. Consider the algebra $K\langle d\rangle$, with the generators denoted by $\left\{x_{j k}: j, k=1, \ldots, d\right\}$. Let $q: K\langle d\rangle \rightarrow \operatorname{Pol}\left(U_{d}^{+}\right)$be the quotient map and set $\rho^{\prime}=\rho \circ q, \eta^{\prime}:=\eta \circ q$. Then $\rho^{\prime}$ is a representation of $K\langle d\rangle$ and $\eta^{\prime}$ is a $\rho^{\prime}$ - $\epsilon$-cocycle on $K\langle d\rangle$. It follows from Theorem 1.10 that the map defined by $(j, k=1, \ldots, d)$

$$
\psi^{\prime}\left(x_{j k}\right)=-\frac{1}{2} \widetilde{B}_{j k}
$$

extends to a generating functional on $K\langle d\rangle$ corresponding to the cocycle $\eta^{\prime}$. We aim to define a functional $\psi: \operatorname{Pol}\left(U_{d}^{+}\right) \rightarrow \mathbb{C}$ by $\psi(q(x)):=\psi^{\prime}(x)$ for $x \in K\langle d\rangle$. According to Lemma 1.11 $\psi$ will be well-defined provided we have for all $j, k=1, \ldots, d$,

$$
\psi^{\prime}\left(\sum_{p=1}^{d} x_{j p}^{*} x_{k p}\right)=0=\psi^{\prime}\left(\sum_{p=1}^{d} x_{p j} x_{p k}^{*}\right) .
$$

Since $\overline{\tilde{B}_{j k}}=\tilde{B}_{k j}$, and by the assumption $\tilde{B}_{k j}=B_{k j}$ we have

$$
\sum_{p=1}^{d} \psi^{\prime}\left(x_{j p}^{*} x_{k p}\right)=\overline{\psi^{\prime}\left(x_{j k}\right)}+\psi^{\prime}\left(x_{k j}\right)+B_{j k}=-\widetilde{B}_{k j}+B_{j k}=0,
$$

and similarly

$$
\sum_{p=1}^{d} \psi^{\prime}\left(x_{p j} x_{p k}^{*}\right)=\psi^{\prime}\left(x_{k j}\right)+\overline{\psi^{\prime}\left(x_{j k}\right)}+\sum_{p=1}^{d}\left\langle\eta\left(x_{p j}^{*}\right), \eta\left(x_{p k}^{*}\right)\right\rangle=-\widetilde{B}_{k j}+\sum_{p=1}^{d}\left\langle\eta\left(x_{p j}^{*}\right), \eta\left(x_{p k}^{*}\right)\right\rangle .
$$

It remains to use Lemma 3.1 and the assumption that $\widetilde{B}=B^{t}$ to conclude that the above expression equals zero.

Thus $\psi$ is well-defined and can be easily checked to be a generating functional associated to $\eta$.

Finally note that Theorem 3.3 takes a very simple form for cocycles with values in $\mathbb{C}$.

Corollary 3.4. Let $\rho: \operatorname{Pol}\left(U_{d}^{+}\right) \rightarrow B(\mathbb{C})$ be a representation, and let $\eta: \operatorname{Pol}\left(U_{d}^{+}\right) \rightarrow \mathbb{C}$ be a $\rho$ - $\epsilon$-cocycle. Then $\eta$ admits a generating functional if and only if $\bar{W} W^{t}=V V^{*}$, where $V$ and $W$ are defined as in (3.1) and (3.2). In particular a Gaussian cocycle $\eta: \operatorname{Pol}\left(U_{d}^{+}\right) \rightarrow \mathbb{C}$ admits a generating functional if and only if $V V^{*}=V^{*} V$.

Proof. It suffices to observe that in the case $H=\mathbb{C}$ we have $B=\bar{V} V^{t}$, and $\widetilde{B}=\bar{W} W^{t}$ and apply Theorem 3.3. In the Gaussian case we use further the equality $W=-V^{t}$. 
3.1. $U_{F}^{+}$for non-generic $F$. In this subsection we will show that for non-generic $F$ the quantum group $U_{F}^{+}$does not have any of the properties (GC), (NC) or (LK). Of course to that end it suffices to show that $U_{F}^{+}$does not have Property (LK), but our proof will proceed by first building Gaussian and purely non-Gaussian cocycles without generating functionals.

Theorem 3.5. Let $d \in \mathbb{N}$ and let $F \in G L_{d}(\mathbb{C})$ be a non-generic matrix (so in particular $d \geq 2)$. Then the compact quantum group $U_{F}^{+}$has none of the properties $(G C),(N C)$ and $(L K)$.

Proof. We begin by considering the case of $F=I_{2}$ (so $\mathbb{G}=U_{2}^{+}$), the general case will follow later. In this proof we will denote the canonical generators of $\operatorname{Pol}\left(U_{2}^{+}\right)$by $\left(v_{j k}\right)_{j, k=1}^{2}$.

No property (GC): Proposition 3.2 implies that any matrix $V \in M_{2}(\mathbb{C})$ defines a Gaussian cocycle $\eta_{G}$ on $U_{2}^{+}$by the formula $\eta_{G}\left(v_{j k}\right)=V_{j k}, j, k=1, \ldots, 2$. Corollary 3.4 implies that if $V$ is not normal, then $\eta_{G}$ does not admit a generating functional. Fix the choice of $V=\left(\begin{array}{ll}1 & i \\ 0 & 1\end{array}\right)$.

No property (NC): consider the purely non-Gaussian representation $\gamma$ of $\operatorname{Pol}\left(U_{2}^{+}\right)$on $\mathbb{C}$, given by the matrix $R=-I_{2}$ and the formula

$$
\gamma\left(v_{j k}\right)=R_{j k}, \quad j, k=1, \ldots, 2 .
$$

Note that here again by Proposition 3.2 any matrix $V^{\prime} \in M_{2}(\mathbb{C})$ defines a purely non-Gaussian cocycle $\eta_{N}$ on $\operatorname{Pol}\left(U_{2}^{+}\right)$by the formula $\eta_{N}\left(v_{j k}\right)=V_{j k}^{\prime}, j, k=1, \ldots, 2$. This time however, by the second part of Proposition [3.2, we have $W^{\prime}=\left(V^{\prime}\right)^{t}$. This change does not affect the necessary condition in Proposition 3.2 and again any choice of a non-normal $V^{\prime}$ yields a cocycle $\eta_{N}$ which does not admit a generating functional. Fix the choice of $V^{\prime}=\left(\begin{array}{ll}1 & 0 \\ i & 1\end{array}\right)$.

No property (LK): consider $H=\mathbb{C} \oplus \mathbb{C}, \rho=\epsilon \oplus \gamma$ and $\eta=\eta_{G} \oplus \eta_{N}$ with $\eta_{G}, \eta_{N}$ not admitting generating functionals constructed above. Then the matrix $R$ is of the form $R=\left(\begin{array}{ll}J & 0 \\ 0 & J\end{array}\right)$ with $J=\left(\begin{array}{cc}1 & 0 \\ 0 & -1\end{array}\right)$, and $V=V e_{1}+V^{\prime} e_{2}$. A direct computation using formulae (3.3) and (3.4) shows that we have

$$
B=\left(\begin{array}{ll}
3 & 0 \\
0 & 3
\end{array}\right)=\widetilde{B}
$$

hence, by Theorem 3.3. $\eta$ admits a generating functional. But this functional has no LévyKhintchine decomposition. If it was the case, then $\left(\epsilon, \eta_{G}\right)$ and $\left(\delta, \eta_{N}\right)$ would admit generating functionals, which was shown not to be the case.

General case: We can assume that $F^{*} F$ is of the form $\operatorname{diag}\left[\lambda_{1}, \ldots, \lambda_{d}\right]$, with $\lambda_{1}=\lambda_{2}=1$. The key role will be played by the unital ${ }^{*}$-homomorphism $\pi: \operatorname{Pol}\left(U_{F}^{+}\right) \rightarrow \operatorname{Pol}\left(U_{2}^{+}\right)$given by the formula

$$
\pi\left(u_{j k}\right)=\left\{\begin{array}{lc}
v_{j k} & \text { for } j, k \in\{1,2\} \\
\delta_{j k} 1 & \text { else. }
\end{array}\right.
$$

No property (GC): Define a Gaussian cocycle $\widetilde{\eta}_{G}$ on $\operatorname{Pol}\left(U_{F}^{+}\right)$by the formula $\widetilde{\eta}_{G}:=\eta_{G} \circ \pi$, with $\eta_{G}$ as in the first part of the proof. Suppose that $\widetilde{\eta}_{G}$ admits a generating functional 
$\psi_{G}: \operatorname{Pol}\left(U_{F}^{+}\right) \rightarrow \mathbb{C}$. Recall that the generators of $U_{F}^{+}$satisfy the two following conditions $(j, k=1, \ldots, d)$ :

$$
\sum_{p=1}^{d} u_{j p} u_{k p}^{*}=\delta_{j k} 1, \quad \sum_{p=1}^{d} \frac{\lambda_{p}}{\lambda_{j}} u_{p k} u_{p j}^{*}=\delta_{j k} 1 .
$$

We are only interested in fact in $j, k=1,2$ and apply $\psi_{G}$ to both of the above equalities. It is easy to see that as $\lambda_{1}=\lambda_{2}=1$ and $\widetilde{\eta}_{G}\left(u_{l m}\right)$ vanishes unless $l, m \in\{1,2\}$, this leads to the following equalities $(j, k=1, \ldots, 2)$ :

$$
0=\psi_{G}\left(u_{j k}\right)+\psi_{G}\left(u_{k j}^{*}\right)+\sum_{p=1}^{2}\left\langle\widetilde{\eta}_{G}\left(u_{j p}^{*}\right), \widetilde{\eta}_{G}\left(u_{k p}^{*}\right)\right\rangle
$$

and

$$
0=\psi_{G}\left(u_{j k}\right)+\psi_{G}\left(u_{k j}^{*}\right)+\sum_{p=1}^{2}\left\langle\widetilde{\eta}_{G}\left(u_{p k}^{*}\right), \widetilde{\eta}_{G}\left(u_{p j}^{*}\right)\right\rangle .
$$

Thus in particular we get

$$
\sum_{p=1}^{2}\left\langle\eta_{G}\left(v_{j p}^{*}\right), \eta_{G}\left(v_{k p}^{*}\right)\right\rangle=\sum_{p=1}^{2}\left\langle\eta_{G}\left(v_{p k}^{*}\right), \eta_{G}\left(v_{p j}^{*}\right)\right\rangle .
$$

This however is nothing but the condition for the existence of the generating functional for $\eta_{G}$, which we know not to hold. Thus $\widetilde{\eta}_{G}$ does not admit a generating functional.

No property (NC): we argue as above, defining first a purely non-Gaussian representation $\widetilde{\gamma}$ of $\operatorname{Pol}\left(U_{F}^{+}\right)$on $\mathbb{C}$ via the formula $\widetilde{\gamma}=\gamma \circ \pi$ and then a $\widetilde{\gamma}$ - $\epsilon$-cocycle $\widetilde{\eta}_{N}:=\eta_{N} \circ \pi$, with $\eta_{N}$ as in the first part of the proof. If we suppose that $\widetilde{\eta}_{N}$ admits a generating functional $\psi_{N}: \operatorname{Pol}\left(U_{F}^{+}\right) \rightarrow \mathbb{C}$, then once again we obtain the equality

$$
\sum_{p=1}^{2}\left\langle\eta_{N}\left(v_{j p}^{*}\right), \eta_{N}\left(v_{k p}^{*}\right)\right\rangle=\sum_{p=1}^{2}\left\langle\eta_{N}\left(v_{p k}^{*}\right), \eta_{N}\left(v_{p j}^{*}\right)\right\rangle,
$$

which contradicts the fact that $\eta_{N}$ does not admit a generating functional.

No property $(\mathbf{L K})$ : this is now straightforward. Define a representation $\widetilde{\rho}: \operatorname{Pol}\left(U_{F}^{+}\right) \rightarrow$ $B(\mathbb{C} \oplus \mathbb{C})$ as $\widetilde{\rho}:=\epsilon \oplus \widetilde{\gamma}$ and the $\widetilde{\rho}-\epsilon$-cocycle $\widetilde{\eta}$ as $\widetilde{\eta}_{G}+\widetilde{\eta}_{N}$. By the first part of the proof $\tilde{\eta}$ possesses a generating functional $\widetilde{\psi}=\psi \circ \pi$, where $\psi: \operatorname{Pol}\left(U_{2}^{+}\right)$is the generating functional for $\eta_{G}+\eta_{N}$. Arguing as in the case of $U_{2}^{+}$we see that $\widetilde{\psi}$ does not admit a Lévy-Khintchine decomposition.

3.2. The free orthogonal quantum group $O_{d}^{+}$. We begin from the case $d=2$.

Proposition 3.6. The compact quantum group $\mathrm{O}_{2}^{+}$has properties (GC) and (LK), but not $(N C)$.

Proof. Recall that $\mathrm{O}_{2}^{+} \cong S U_{-1}(2)$. The statements follow from the results from [ScS] (see also $\left[\mathrm{Ske}_{2}\right]$ ). (They can be also easily deduced from Proposition 3.7 and Corollary 3.9 below.)

We shall see that the situation changes for $O_{d}^{+}$with $d \geq 3$. For that we first prove the analogue of Proposition 3.2 in the case of the free orthogonal group. 
Proposition 3.7. Let $H$ be a Hilbert space, and let $\rho: \operatorname{Pol}\left(O_{d}^{+}\right) \rightarrow B(H)$ be a representation, define the matrix $R$ as in the first formula in (3.1) and let $V \in M_{d}(H)$. Then the following conditions are equivalent:

(i) there is a $\rho$ - $\epsilon$-cocycle $\eta$ on $\operatorname{Pol}\left(O_{d}^{+}\right)$satisfying the second formula in (3.1);

$$
\left(R^{*} V\right)^{t}=\bar{R} V^{t}=-V .
$$

Proof. Any representation of $\operatorname{Pol}\left(O_{d}^{+}\right)$lifts canonically to a representation of $\operatorname{Pol}\left(U_{d}^{+}\right)$, simply by composition with the quotient maps, and the same is true for the corresponding cocycles. Thus the condition $\left(R^{*} V\right)^{t}=\bar{R} V^{t}$ from Proposition 3.2 is necessary. Moreover every cocycle on $\operatorname{Pol}\left(O_{d}^{+}\right)$must of course satisfy the conditions $\eta\left(u_{j k}\right)=\eta\left(u_{j k}^{*}\right)$, for $j, k=1, \ldots, d$, which in terms of the proof of Proposition 3.2 means that $W=V$. Hence we must have $-V=-W=$ $\bar{R} V^{t}=\left(R^{*} V\right)^{t}$.

Conversely, if $V$ satisfies (3.6), then - as in the proof of Proposition 3.2 - $\eta$ defined by $V$ vanishes on the relations that define $O_{d}^{+}$and hence it can be extended by linearity and the cocycle property to the whole algebra in question.

Now, we check when a given $\rho$ - $\epsilon$-cocycle on $\operatorname{Pol}\left(O_{d}^{+}\right)$admits a generating functional. We use the same methods as in Theorem 3.3, with the only difference being that now matrices $B$ and $\widetilde{B}$ of (3.3) and (3.4) naturally coincide (and are self-adjoint).

Theorem 3.8. Let $H$ be a Hilbert space, let $\rho: \operatorname{Pol}\left(O_{d}^{+}\right) \rightarrow B(H)$ be a representation, and let $\eta: \operatorname{Pol}\left(O_{d}^{+}\right) \rightarrow H$ be a $\rho$ - $\epsilon$-cocycle. Then $\eta$ admits a generating functional if and only if the matrix $B$ defined in (3.3) is symmetric, which is the case if and only if $B$ has real entries.

The special case of Theorem 3.8 for $\mathbb{C}$-valued cocycles, an analogue of Corrolary 3.4, for the free orthogonal group takes the following form.

Corollary 3.9. Let $\rho: \operatorname{Pol}\left(O_{d}^{+}\right) \rightarrow B(\mathbb{C})$ be a representation, and let $\eta: \operatorname{Pol}\left(O_{d}^{+}\right) \rightarrow \mathbb{C}$ be a $\rho-\epsilon$-cocycle. Then $\eta$ admits a generating functional if and only if $V V^{*}$ has real entries, where $V$ is defined as in (3.1).

We are ready to show the main result of this section.

Theorem 3.10. Let $d \in \mathbb{N}, d \geq 3$. Then the compact quantum group $O_{d}^{+}$has none of the properties $(G C)$, (NC) and $(L K)$.

Proof. No property (GC): consider the block matrix

$$
V=\left(\begin{array}{cc}
V_{1} & 0 \\
0 & 0
\end{array}\right)
$$

with

$$
V_{1}=\left(\begin{array}{ccc}
0 & 1 & 1 \\
-1 & 0 & i \\
-1 & -i & 0
\end{array}\right)
$$

Proposition 3.7 implies, as $V=-V^{t}$, that the formula (3.1) defines a $\mathbb{C}$-valued Gaussian cocycle on $\operatorname{Pol}\left(O_{d}^{+}\right)$. On the other hand, as

$$
V_{1} V_{1}^{*}=\left(\begin{array}{ccc}
2 & -i & i \\
i & 2 & 1 \\
-i & 1 & 2
\end{array}\right),
$$


Corollary 3.9 shows that this cocycle does not admit a generating functional.

No property (NC): consider the purely non-Gaussian representation $\gamma$ of $\operatorname{Pol}\left(O_{d}^{+}\right)$on $\mathbb{C}$, given by the matrix

and the formula

$$
R=\left(\begin{array}{cccc}
-1 & 0 & 0 & \ldots \\
0 & 0 & 1 & \ldots \\
0 & 1 & 0 & \ldots \\
\vdots & \vdots & \vdots & \ddots
\end{array}\right)
$$

$$
\gamma\left(u_{j k}\right)=R_{j k}, \quad j, k=1, \ldots, d .
$$

Further consider the block matrix

$$
V^{\prime}=\left(\begin{array}{cc}
V_{1}^{\prime} & 0 \\
0 & 0
\end{array}\right)
$$

with

$$
V_{1}^{\prime}=\left(\begin{array}{ccc}
i & 1 & -1 \\
1 & 0 & 0 \\
-1 & 0 & 0
\end{array}\right)
$$

Proposition 3.7 implies, as $V=-\bar{R} V^{t}=-\left(R^{*} V\right)^{t}$, that the formula (3.1) defines a $\mathbb{C}$-valued $\gamma$ - $\epsilon$-cocycle on $\operatorname{Pol}\left(O_{d}^{+}\right)$. On the other hand, as

$$
V_{1}^{\prime}\left(V_{1}^{\prime}\right)^{*}=\left(\begin{array}{ccc}
3 & i & -i \\
-i & 1 & -1 \\
i & -1 & 1
\end{array}\right),
$$

once again Corollary 3.9 shows that this cocycle does not admit a generating functional.

No property (LK): The proof now proceeds as in Theorem 3.5, we consider the direct sum of the representations and cocycles in the last two cases, and observe that the corresponding matrix $B$ of (3.3) for the resulting cocycle is the block-diagonal matrix of the form

$$
B=\left(\begin{array}{cc}
B_{1} & 0 \\
0 & 0
\end{array}\right)
$$

with

$$
B_{1}=V_{1} V_{1}^{*}+V_{1}^{\prime}\left(V_{1}^{\prime}\right)^{*}=\left(\begin{array}{ccc}
5 & 0 & 0 \\
0 & 3 & 0 \\
0 & 0 & 3
\end{array}\right) .
$$

Thus by Theorem 3.8 the last cocycle admits a generating functional. As before, the earlier parts of the proof imply that this generating functional cannot admit a Lévy-Khintchine decomposition.

The result above can be easily adopted, using techniques similar to these in Theorem 3.5, to show that the quantum group $O_{F}^{+}$with $F$ of the form

$$
\left(\begin{array}{ccc}
0 & D & 0 \\
D^{-1} & 0 & 0 \\
0 & 0 & I_{k}
\end{array}\right)
$$

with $k \geq 3$, does not have any of the properties (GC), (NC) and (LK). The case of general $O_{F}^{+}$, with $F \in G L_{d}(\mathbb{C}), d \geq 3$, seems however beyond our reach for the moment. 
3.3. Further remarks. Methods developed in this section lead to certain further observations.

Recall first that for any compact quantum group $\mathbb{G}$ a cocycle $\eta: \operatorname{Pol}(\mathbb{G}) \rightarrow H$ is said to be real ( Kye $)$ if for any $a, b \in \operatorname{Pol}(\mathbb{G})$

$$
\langle\eta(a), \eta(b)\rangle=\left\langle\eta\left(S(b)^{*}\right), \eta\left(S\left(a^{*}\right)\right)\right\rangle
$$

where $S$ denotes the antipode of $\operatorname{Pol}(\mathbb{G})$. In DFKS] we showed that any real cocycle admits a generating functional. The following example shows that the reality of $\eta$ is not necessary; it also provides an example of noncommutative Gaussian process on $O_{4}^{+}$.

Example 3.11. Consider the following matrix $V \in M_{4}\left(\mathbb{C}^{2}\right)$ :

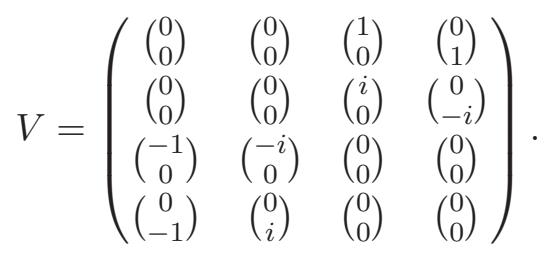

Proposition 3.7 shows that the matrix $V$, as satisfying the condition $V=-V^{t}$, defines a Gaussian cocycle $\eta: \operatorname{Pol}\left(O_{4}^{+}\right) \rightarrow \mathbb{C}^{2}$ via the formula (3.1). A straightforward computation shows that then the corresponding matrix $B$, defined as in (3.3) is equal to $2 I_{4}$. Thus Theorem 3.8 shows that $\eta$ admits a generating functional $\psi: \operatorname{Pol}\left(O_{4}^{+}\right) \rightarrow \mathbb{C}$. On the other hand $\eta$ is not real, as we have for example

$$
\left\langle\eta\left(u_{23}\right), \eta\left(u_{31}\right)\right\rangle=i \neq-i=\left\langle\eta\left(u_{13}\right), \eta\left(u_{32}\right)\right\rangle=\left\langle\eta\left(S\left(u_{31}\right)^{*}\right), \eta\left(S\left(u_{23}^{*}\right)\right)\right\rangle .
$$

We can also check that the functional $\psi$ constructed for $\eta$ as in Theorem 3.8 is non-tracial: indeed, we have

$$
\psi\left(u_{23} u_{31}\right)=\left\langle\eta\left(u_{23}\right), \eta\left(u_{31}\right)\right\rangle \neq\left\langle\eta\left(u_{31}\right), \eta\left(u_{23}\right)\right\rangle=\psi\left(u_{31} u_{23}\right) .
$$

It was shown in [FKS, Proposition 5.7] that a Gaussian Lévy process is commutative if and only its generating functional is a trace. So this shows that the Gaussian Lévy process associated to this cocycle is indeed not commutative, as we claimed above.

Using the techniques of this section one can show that the half-liberated orthogonal quantum group $O_{d}^{*}$ (see $[\mathrm{BaV}]$ ) for $d \geq 3$ does not have properties (GC) or (NC).

Finally let $\mathbb{G}$ be a compact quantum group with a compact quantum subgroup $\mathbb{H}$ (i.e. we have a surjective Hopf *-algebra homomorphism $\pi: \operatorname{Pol}(\mathbb{G}) \rightarrow \operatorname{Pol}(\mathbb{H}))$. It is easy to see that any generating functional (any cocycle, or any Schürmann triple) on $\mathbb{H}$ can be transported in an obvious way to $\mathbb{G}$ (the fact we used several times above). However the relationship between properties $(\mathrm{GC}),(\mathrm{NC})$ or $(\mathrm{LK})$ for the pair $(\mathbb{G}, \mathbb{H})$ are far from straightforward. Indeed, if we consider the sequence of quantum subgroups $S U_{q}(2) \subset S U_{q}(3) \subset U_{F}^{+}$(with $F$ the $3 \times 3$ matrix from Example 1.4), we see from the results of the last two sections that $S U_{q}(2)$ has $(\mathrm{GC}), S U_{q}(3)$ does not have $(\mathrm{GC})$, and $U_{3}^{+}(F)$ has $(\mathrm{GC})$ again. So far we do not know any case where $\mathbb{G}$ would have property $(\mathrm{LK})$ and its quantum subgroup $\mathbb{H}$ would fail to have this property.

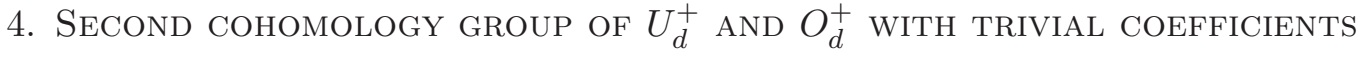

It is well-known that the Lévy-Khintchine decomposition problem for a given unitary quotient algebra $A$ is closely related to the computation of the second Hochschild cohomology 
group of $A$ with trivial coefficients (see for example [FGT]). In this section we show certain consequences of our earlier results for the corresponding second Hochschild cohomology groups for $\operatorname{Pol}\left(O_{d}^{+}\right)$and $\operatorname{Pol}\left(U_{d}^{+}\right)$.

Let us briefly recall the definitions (we refer to [FGT] and to the survey [Bica for more details). Given a unital $*$-algebra $A$ with a character $\epsilon$ (e.g. a unitary quotient algebra), any representation $\rho: A \rightarrow B(H)$ on a Hilbert space $H$ allows to view $H$ as an $A$-bimodule with the left and right actions $a . z . b=\rho(a) \epsilon(b) z$ for $a, b \in A$ and $v \in H$. As a special case, we can consider $\mathbb{C}$ as an $A$-bimodule with the 'trivial' left and right actions, i.e. $a . z . b=\epsilon(a) \epsilon(b) z$ for $a, b \in A$ and $z \in \mathbb{C}$.

For each $n \in \mathbb{N}$ the associated coboundary operator $\partial^{n-1}: L\left(A^{\otimes(n-1)} ; H\right) \rightarrow L\left(A^{\otimes n} ; H\right)$ (where we write $A^{\otimes 0}:=\mathbb{C}$ ) is determined by the formula

$$
\begin{aligned}
\left(\partial^{n-1} \phi\right)\left(a_{1} \otimes \ldots \otimes a_{n}\right) & =\rho\left(a_{1}\right) \phi\left(a_{2} \otimes \ldots \otimes a_{n}\right)+\sum_{j=1}^{n-1}(-1)^{j} \phi\left(a_{1} \otimes \ldots \otimes\left(a_{j} a_{j+1}\right) \otimes \ldots \otimes a_{n}\right) \\
& +(-1)^{n} \phi\left(a_{1} \otimes \ldots \otimes a_{n-1}\right) \epsilon\left(a_{n}\right)
\end{aligned}
$$

for $\phi \in L\left(A^{\otimes(n-1)} ; H\right), a_{1}, \ldots, a_{n} \in A$. We naturally have $\partial^{n} \circ \partial^{n-1}=0$. We define further the vector space of $n$-cocycles $Z^{n}\left(A,{ }_{\rho} H_{\epsilon}\right)=\left\{\phi \in L\left(A^{\otimes n} ; H\right): \partial^{n} \phi=0\right\}$, the vector space of $n$-coboundaries $B^{n}\left(A,{ }_{\rho} H_{\epsilon}\right)=\left\{\partial^{n-1} \psi: \psi \in L\left(A^{\otimes(n-1)}, H\right)\right\}$, and the nth-Hochschild cohomology group

$$
H^{n}\left(A,{ }_{\rho} H_{\epsilon}\right)=Z^{n}\left(A,{ }_{\rho} H_{\epsilon}\right) / B^{n}\left(A,{ }_{\rho} H_{\epsilon}\right) .
$$

The $n$ th-Hochschild cohomology group with trivial coefficients is the special case

$$
H^{n}\left(A,{ }_{\epsilon} \mathbb{C}_{\epsilon}\right)=Z^{n}\left(A,{ }_{\epsilon} \mathbb{C}_{\epsilon}\right) / B^{n}\left(A,{ }_{\epsilon} \mathbb{C}_{\epsilon}\right) .
$$

The terrminology 'trivial coefficients' refers to the fact that we could replace $\mathbb{C}$ above by any $A$-bimodule; and further in the case $A=\mathbb{C}[\Gamma]$ the $A$-bimodule ${ }_{\epsilon} \mathbb{C}_{\epsilon}$ can be viewed equivalently as a trivial $\Gamma$-bimodule.

Note that if $A$ is a unitary quotient algebra, then by construction $H^{n}\left(A,{ }_{\rho} H_{\epsilon}\right)$ is in fact a vector space over $\mathbb{C}$. Moreover $H^{0}\left(A,{ }_{\epsilon} \mathbb{C}_{\epsilon}\right) \cong \mathbb{C}$ and $H^{1}\left(A,{ }_{\epsilon} \mathbb{C}_{\epsilon}\right)$ coincides with the space of $\mathbb{C}$-valued Gaussian cocycles defined in Section 1.2 .

To simplify the notation for a compact matrix quantum group $\mathbb{G}$ and $n \in \mathbb{N}$ we will simply write $H^{n}(\mathbb{G})$ for $H^{n}\left(\operatorname{Pol}(\mathbb{G}),{ }_{\epsilon} \mathbb{C}_{\epsilon}\right)$; and similarly $Z^{n}(\mathbb{G})$ and $B^{n}(\mathbb{G})$ for $Z^{n}\left(\operatorname{Pol}(\mathbb{G}),{ }_{\epsilon} \mathbb{C}_{\epsilon}\right)$, $B^{n}\left(\operatorname{Pol}(\mathbb{G}), \mathbb{C}_{\epsilon}\right)$.

Then it follows from Propositions 3.2 and 3.7 respectively, that for each $d \in \mathbb{N}$ we have

$$
H^{1}\left(U_{d}^{+}\right) \cong M_{d}(\mathbb{C}), \quad \text { and } \quad H^{1}\left(O_{d}^{+}\right) \cong\left\{V \in M_{d}(\mathbb{C}): V^{t}=-V\right\} \cong \mathbb{C}^{\frac{d(d-1)}{2}}
$$

(see also [CHT]). Similarly the proof of Theorem 2.1 and arguments similar to those in the proof of Proposition 2.3 show that for $d \in \mathbb{N}$ and $F \in G L_{d}(\mathbb{C})$ generic we have $H^{1}\left(U_{F}^{+}\right) \cong \mathbb{C}^{d}$ and for $q \in(0,1), d \geq 2$ we have $H^{1}\left(S U_{q}(d)\right) \cong \mathbb{C}^{d-1}$.

If for a given compact matrix quantum group $\mathbb{G}$ the space $H^{2}(\mathbb{G})$ is trivial, then $\mathbb{G}$ has all the properties $(\mathrm{GC}),(\mathrm{NC})$ and $(\mathrm{LK})$, see $[\mathrm{FGT}$. Thus the results of the last section imply that $H^{2}\left(U_{d}^{+}\right)$is non-trivial for $d \geq 2$ and $H^{2}\left(O_{d}^{+}\right)$is non-trivial for $d \geq 3$.

We will now recall a few general facts which will be helpful in computing $H^{2}(\mathbb{G})$ in the rest of this section. First note that one can restrict attention to normalised 2-cocycles, i.e. those $c \in Z^{2}(\mathbb{G})$ which satisfy the condition $c(1 \otimes 1)=0$. Indeed, given a general cocycle $c \in Z^{2}(\mathbb{G})$ one can always pass to the normalised 2-cocycle $c^{\prime}:=c-c(1 \otimes 1) \partial \epsilon$, which naturally yields 
the same class in $H^{2}(\mathbb{G})$. Furthermore it is easy to check that if a 2-cocycle $c$ is normalised, then in fact $c(1 \otimes a)=c(a \otimes 1)=0$ for all $a \in \operatorname{Pol}(\mathbb{G})$.

The following result is Lemma 5.4 of $[\mathrm{BFG}]$.

Lemma 4.1. Suppose that $(A, \epsilon)$ is a unital ${ }^{*}$-algebra with a character, $\psi: A \rightarrow \mathbb{C}$ is a linear functional with $\psi(1)=0$ and $c \in Z^{2}\left(A, \mathbb{C}_{\epsilon}\right)$ is a normalised 2-cocycle. Define the map $T: A \rightarrow \operatorname{End}(\mathbb{C} \oplus \operatorname{ker} \epsilon \oplus \mathbb{C})$ via the formula

$$
T(a)=\left(\begin{array}{ccc}
\epsilon(a) & c(a \otimes-) & -\psi(a) \\
0 & a \cdot- & a-\epsilon(a) 1 \\
0 & 0 & \epsilon(a)
\end{array}\right), \quad a \in A .
$$

Then the map $T$ is a homomorphism if and only if $c=\partial \psi$.

The next lemma follows from an elementary computation (see for example the proof of Proposition 3.1 in [FGT] $)$.

Lemma 4.2. Let $A$ be a unitary quotient algebra and let $\rho: A \rightarrow B(H)$ be a representation of $A$ on a Hilbert space $H$. If $\eta_{1}, \eta_{2}: A \rightarrow H$ are $\rho$ - $\epsilon$-cocycles on $A$, then the formula

$$
K\left(\eta_{1}, \eta_{2}\right)(a \otimes b):=\left\langle\eta_{1}\left(a^{*}\right), \eta_{2}(b)\right\rangle, \quad a, b \in A
$$

determines a 2-cocycle $K\left(\eta_{1}, \eta_{2}\right)$ in $Z_{2}\left(A,{ }_{\epsilon} \mathbb{C}_{\epsilon}\right)$.

4.1. Computing $H^{2}\left(U_{d}^{+}\right)$. Let $d \in \mathbb{N}$. We will compute now the group $H^{2}\left(U_{d}^{+}\right)$. Begin with the following lemma, describing properties of arbitrary normalised 2-cocycles in $Z^{2}\left(U_{d}^{+}\right)$.

Lemma 4.3. Let $c \in Z^{2}\left(U_{d}^{+}\right)$be normalised. Then for all $j, k \in\{1, \ldots, d\}$ we have relations

$$
\begin{aligned}
& \sum_{p=1}^{d} c\left(u_{p j}^{*} \otimes u_{p k}\right)=\sum_{p=1}^{d} c\left(u_{j p} \otimes u_{k p}^{*}\right), \\
& \sum_{p=1}^{d} c\left(u_{j p}^{*} \otimes u_{k p}\right)=\sum_{p=1}^{d} c\left(u_{p j} \otimes u_{p k}^{*}\right) .
\end{aligned}
$$

Proof. Fix $j, k \in\{1, \ldots, d\}$ and apply $\partial c$ to $\sum_{p, r=1}^{d} u_{j p} \otimes u_{r p}^{*} \otimes u_{r k}$ to obtain

$$
\begin{aligned}
0 & =\sum_{p, r=1}^{d} \partial c\left(u_{j p} \otimes u_{r p}^{*} \otimes u_{r k}\right)= \\
& =\sum_{p, r=1}^{d}\left[\epsilon\left(u_{j p}\right) c\left(u_{r p}^{*} \otimes u_{r k}\right)-c\left(u_{j p} u_{r p}^{*} \otimes u_{r k}\right)+c\left(u_{j p} \otimes u_{r p}^{*} u_{r k}\right)-c\left(u_{j p} \otimes u_{r p}^{*}\right) \epsilon\left(u_{r k}\right)\right] \\
& =\sum_{r=1}^{d} c\left(u_{r j}^{*} \otimes u_{r k}\right)-\sum_{r=1}^{d} c\left(\sum_{p=1}^{d} u_{j p} u_{r p}^{*} \otimes u_{r k}\right)+\sum_{p=1}^{d} c\left(u_{j p} \otimes \sum_{r=1}^{d} u_{r p}^{*} u_{r k}\right)-\sum_{p=1}^{d} c\left(u_{j p} \otimes u_{k p}^{*}\right) \\
& \stackrel{(*)}{=} \sum_{r=1}^{d} c\left(u_{r j}^{*} \otimes u_{r k}\right)-\sum_{r=1}^{d} c\left(1 \otimes u_{r k}\right)+\sum_{p=1}^{d} c\left(u_{j p} \otimes 1\right)-\sum_{p=1}^{d} c\left(u_{j p} \otimes u_{k p}^{*}\right) \\
& =\sum_{r=1^{d}} c\left(u_{r j}^{*} \otimes u_{r k}\right)-\sum_{p=1^{d}} c\left(u_{j p} \otimes u_{k p}^{*}\right),
\end{aligned}
$$


where in $(*)$ we used the unitarity relations $u^{*} u=u u^{*}=I$. This shows (4.2).

Similarly applying $\partial c$ to the $\operatorname{sum} \sum_{p, r=1}^{d} u_{p j} \otimes u_{p r}^{*} \otimes u_{j r}$ and using the fact that $\bar{u} u^{t}=u^{t} \bar{u}=I$ yields (4.3).

The next result allows us to characterise coboundaries in $Z^{2}\left(U_{d}^{+}\right)$.

Lemma 4.4. A 2-cocycle $c \in Z^{2}\left(U_{d}^{+}\right)$is a coboundary if and only if for each $j, k \in\{1, \ldots, d\}$

$$
\sum_{p=1}^{d} c\left(u_{p j}^{*} \otimes u_{p k}\right)=\sum_{p=1}^{d} c\left(u_{k p}^{*} \otimes u_{j p}\right) .
$$

Proof. Note first that we can assume we are dealing with normalised 2-cocycles; the passage $c \mapsto c-\lambda \partial \epsilon$ for $\lambda \in \mathbb{C}$ does not affect neither being a coboundary, nor satisfying the equalities in (4.4).

$(\Rightarrow)$ Assume that $c \in Z^{2}\left(U_{d}^{+}\right)$is a normalised coboundary, so that there exists a functional $\psi: \operatorname{Pol}\left(U_{d}^{+}\right) \rightarrow \mathbb{C}$ such that $\psi(1)=0$ and $c=\partial \psi$. Then for every $j, k \in\{1, \ldots, d\}$

$$
\begin{aligned}
\sum_{p=1}^{d} c\left(u_{p j}^{*} \otimes u_{p k}\right) & =\sum_{p=1}^{d} \partial \psi\left(u_{p j}^{*} \otimes u_{p k}\right)=\sum_{p=1}^{d}\left(\psi\left(u_{p j}^{*}\right) \epsilon\left(u_{p k}\right)-\psi\left(u_{p j}^{*} u_{p k}\right)+\epsilon\left(u_{p j}^{*}\right) \psi\left(u_{p k}\right)\right) \\
& =\psi\left(u_{k j}^{*}\right)-\psi\left(\sum_{p=1}^{d} u_{p j}^{*} u_{p k}\right)+\psi\left(u_{j k}\right) \stackrel{u^{*}}{=}=I\left(u_{k j}^{*}\right)+\psi\left(u_{j k}\right),
\end{aligned}
$$

and similarly

$$
\begin{aligned}
\sum_{p=1}^{d} c\left(u_{k p}^{*} \otimes u_{j p}\right) & =\sum_{p=1}^{d} \partial \psi\left(u_{k p}^{*} \otimes u_{j p}\right)=\sum_{p=1}^{d}\left(\psi\left(u_{k p}^{*}\right) \epsilon\left(u_{j p}\right)-\psi\left(u_{k p}^{*} u_{j p}\right)+\epsilon\left(u_{k p}^{*}\right) \psi\left(u_{j p}\right)\right) \\
& =\psi\left(u_{k j}^{*}\right)-\psi\left(\sum_{p=1}^{d} u_{k p}^{*} u_{j p}\right)+\psi\left(u_{j k}\right) \stackrel{\bar{u} u^{t}=I}{=} \psi\left(u_{k j}^{*}\right)+\psi\left(u_{j k}\right) .
\end{aligned}
$$

This shows that (4.4) holds.

$(\Leftarrow)$ Let then $c \in Z^{2}\left(U_{d}^{+}\right)$be normalised and satisfying (4.4). Set $\psi(1)=0$,

$$
\psi\left(u_{j k}\right)=\psi\left(u_{k j}^{*}\right):=\frac{1}{2} \sum_{p=1}^{d} c\left(u_{p j}^{*} \otimes u_{p k}\right), \quad j, k \in\{1, \ldots, d\} .
$$

We are going to prove that the map $T=T_{c, \psi}$ defined via the prescription (4.1) for $a \in$ $\left\{1, u_{j k}, u_{j k}^{*}: j, k=1, \ldots d\right\}$ extends to a homomorphism $\tilde{T}: \operatorname{Pol}\left(U_{d}^{+}\right) \rightarrow \operatorname{End}(\mathbb{C} \oplus \operatorname{ker} \epsilon \oplus \mathbb{C})$. For that it suffices to check that elements $t_{j k}:=T_{c, \psi}\left(u_{j k}\right), j, k \in\{1, \ldots, d\}$ satisfy the relations that define the algebra $\operatorname{Pol}\left(U_{d}^{+}\right)$. Then we will be able to define the functional $\tilde{\psi}: \operatorname{Pol}\left(U_{d}^{+}\right) \rightarrow \mathbb{C}$ via the formula $\tilde{\psi}(a):=-(\tilde{T}(a))_{13}, a \in \operatorname{Pol}\left(U_{d}^{+}\right)$and finally conclude by Lemma 4.1 that $\partial \tilde{\psi}=c$, so that in particular $c$ is a coboundary. 
We first check that the matrix $t:=\left(t_{j k}\right)_{j, k=1}^{d}$ satisfies the condition $t^{*} t=I$, i.e. we have for each $j, k \in\{1, \ldots, d\}$ the equality $\sum_{p=1}^{d} t_{p j}^{*} t_{p k}=\delta_{j k} I$. This is indeed the case, as

$$
\begin{aligned}
& \sum_{p=1}^{d} t_{p j}^{*} t_{p k}=\sum_{p=1}^{d} T\left(u_{p j}^{*}\right) T\left(u_{p k}\right) \\
& =\sum_{p=1}^{d}\left(\begin{array}{ccc}
\epsilon\left(u_{p j}^{*}\right) & c\left(u_{p j}^{*} \otimes-\right) & -\psi\left(u_{p j}^{*}\right) \\
0 & u_{p j}^{*} \cdot- & u_{p j}^{*}-\epsilon\left(u_{p j}^{*}\right) 1 \\
0 & 0 & \epsilon\left(u_{p j}^{*}\right)
\end{array}\right)\left(\begin{array}{ccc}
\epsilon\left(u_{p k}\right) & c\left(u_{p k} \otimes-\right) & -\psi\left(u_{p k}\right) \\
0 & u_{p k} \cdot- & u_{p k}-\epsilon\left(u_{p k}\right) 1 \\
0 & 0 & \epsilon\left(u_{p k}\right)
\end{array}\right) \\
& =\left(\begin{array}{cccc}
\sum_{p=1}^{d} \epsilon\left(u_{p j}^{*}\right) \epsilon\left(u_{p k}\right) & (\star) & (\star \star) \\
0 & \sum_{p=1}^{d} u_{p j}^{*} u_{p k} \cdot- & \sum_{p=1}^{d}\left(u_{p j}^{*}\left[u_{p k}-\epsilon\left(u_{p k}\right) 1\right]+\left[u_{p j}^{*}-\epsilon\left(u_{p j}^{*}\right) 1\right] \epsilon\left(u_{p k}\right)\right) \\
0 & 0 & \sum_{p=1}^{d} \epsilon\left(u_{p j}^{*}\right) \epsilon\left(u_{p k}\right)
\end{array}\right)
\end{aligned}
$$

and further

$$
\begin{aligned}
& \sum_{p=1}^{d} t_{p j}^{*} t_{p k}=\left(\begin{array}{ccc}
\epsilon\left(\sum_{p=1}^{d} u_{p j}^{*} u_{p k}\right) & (\star) \\
0 & \sum_{p=1}^{d} u_{p j}^{*} u_{p k} \cdot- & \sum_{p=1}^{d}\left(\left[u_{p j}^{*} u_{p k}-\epsilon\left(u_{p j}^{*}\right) 1\right] \epsilon\left(u_{p k}\right)\right) \\
0 & 0 & \epsilon\left(\sum_{p=1}^{d} u_{p j}^{*} u_{p k}\right)
\end{array}\right) \\
& =\left(\begin{array}{ccc}
\delta_{j k} & (\star) & (\star \star) \\
0 & \delta_{j k} 1 \cdot- & 0 \\
0 & 0 & \delta_{j k}
\end{array}\right) .
\end{aligned}
$$

It remains then to check that $(\star)=0$ and that $(\star \star)=0$. The first fact holds due to the fact that $c$ is a normalised cocycle and that the arguments are taken from ker $\epsilon$, since

$$
(\star)=\sum_{p=1}^{d}\left(\epsilon\left(u_{p j}^{*}\right) c\left(u_{p k} \otimes-\right)+c\left(u_{p j}^{*} \otimes u_{p k} \cdot-\right)\right)=\sum_{p=1}^{d}\left(c\left(u_{p j}^{*} u_{p k} \otimes-\right)+c\left(u_{p j}^{*} \otimes u_{p k}\right) \epsilon(-)\right)=0 .
$$

The second formula holds true because of (4.5):

$$
\begin{aligned}
(\star \star) & =\sum_{p=1}^{d}\left(-\epsilon\left(u_{p j}^{*}\right) \psi\left(u_{p k}\right)+c\left(u_{p j}^{*} \otimes\left[u_{p k}-\epsilon\left(u_{p k}\right) 1\right]\right)-\psi\left(u_{p j}^{*}\right) \epsilon\left(u_{p k}\right)\right) \\
& =-\psi\left(u_{j k}\right)+\sum_{p=1}^{d} c\left(u_{p j}^{*} \otimes u_{p k}\right)-\psi\left(u_{k j}^{*}\right)=-2 \cdot \frac{1}{2} \sum_{r=1}^{d} c\left(u_{r j}^{*} \otimes u_{r k}\right)+\sum_{p=1}^{d} c\left(u_{p j}^{*} \otimes u_{p k}\right)=0 .
\end{aligned}
$$

The fact that $t t^{*}=I$ follows in the same way - note that we have not yet used the equality (4.4). 
Next, we verify that $t^{t} \bar{t}=I$, i.e. for each $j, k \in\{1, \ldots, d\}$ we have $\sum_{p=1}^{d} t_{p j} t_{p k}^{*}=\delta_{j k} I$. Indeed, we have

$$
\begin{aligned}
\sum_{p=1}^{d} t_{p j} t_{p k}^{*} & =\sum_{p=1}^{d} T\left(u_{p j}\right) T\left(u_{p k}^{*}\right) \\
& =\sum_{p=1}^{d}\left(\begin{array}{ccc}
\epsilon\left(u_{p j}\right) & c\left(u_{p j} \otimes-\right) & -\psi\left(u_{p j}\right) \\
0 & u_{p j} \cdot- & u_{p j}-\epsilon\left(u_{p j}\right) 1 \\
0 & 0 & \epsilon\left(u_{p j}\right)
\end{array}\right)\left(\begin{array}{ccc}
\epsilon\left(u_{p k}^{*}\right) & c\left(u_{p k}^{*} \otimes-\right) & -\psi\left(u_{p k}^{*}\right) \\
0 & u_{p k}^{*} \cdot- & u_{p k}^{*}-\epsilon\left(u_{p k}^{*}\right) 1 \\
0 & (\diamond) & \epsilon\left(u_{p k}^{*}\right)
\end{array}\right) \\
& =\left(\begin{array}{ccc}
\epsilon\left(\sum_{p=1}^{d} u_{p j} u_{p k}^{*}\right) \\
0 & \sum_{p=1}^{d} u_{p j} u_{p k}^{*} \cdot- & \sum_{p=1}^{d}\left(\begin{array}{cc}
\left.u_{p j} u_{p k}^{*}-\epsilon\left(u_{p j}\right) \epsilon\left(u_{p k}^{*}\right) 1\right) \\
0
\end{array}\right. \\
\quad= & 0 & \epsilon\left(\sum_{p=1}^{d} u_{p j} u_{p k}^{*}\right)
\end{array}\right) \\
& \left(\begin{array}{ccc}
\delta_{j k} & (\diamond) & (\diamond \diamond) \\
0 & \delta_{j k} 1 \cdot- & 0 \\
0 & 0 & \delta_{j k}
\end{array}\right)
\end{aligned}
$$

and, as above,

$(\diamond)=\sum_{p=1}^{d}\left(\epsilon\left(u_{p j}\right) c\left(u_{p k}^{*} \otimes-\right)+c\left(u_{p j} \otimes u_{p k}^{*} \cdot-\right)\right)=\sum_{p=1}^{d}\left(c\left(u_{p j} u_{p k}^{*} \otimes-\right)+c\left(u_{p j} \otimes u_{p k}^{*}\right) \epsilon(-)\right)=0$.

and

$$
\begin{aligned}
(\diamond) & =\sum_{p=1}^{d}\left(-\epsilon\left(u_{p j}\right) \psi\left(u_{p k}^{*}\right)+c\left(u_{p j} \otimes\left[u_{p k}^{*}-\epsilon\left(u_{p k}^{*}\right) 1\right]\right)-\psi\left(u_{p j}\right) \epsilon\left(u_{p k}^{*}\right)\right) \\
& =-\psi\left(u_{j k}^{*}\right)+\sum_{p=1}^{d} c\left(u_{p j} \otimes u_{p k}^{*}\right)-\psi\left(u_{k j}\right) \stackrel{(4.5)}{=}-2 \cdot \frac{1}{2} \sum_{p=1}^{d} c\left(u_{p k}^{*} \otimes u_{p j}\right)+\sum_{p=1}^{d} c\left(u_{p j} \otimes u_{p k}^{*}\right) .
\end{aligned}
$$

But, due to (4.4) and (4.3), we see that

$$
(\diamond \diamond)=\sum_{p=1}^{d} c\left(u_{p k} \otimes u_{p j}^{*}\right)-\sum_{p=1}^{d} c\left(u_{p j}^{*} \otimes u_{p k}\right) \stackrel{\sqrt[4.3]{=}}{=} \sum_{p=1}^{d} c\left(u_{k p}^{*} \otimes u_{j p}\right)-\sum_{p=1}^{d} c\left(u_{p j}^{*} \otimes u_{p k}\right) \stackrel{4.40}{=} 0 .
$$

Finally the equality $\bar{t} t=I$ can be verified in the same manner.

Before we pass to the main result of this subsection we note another interesting fact which can be deduced from the proof of the last two lemmas. Indeed, it follows from the proofs of Lemma 4.3 and Lemma 4.4 that any 2-cocycle on the Brown-Glockner-von Waldenfels algebra $K\langle d\rangle$ (for any $d \in \mathbb{N}$ ) is a coboundary. Indeed, any 2-cocycle on $K\langle d\rangle$ satisfies the condition (4.2), since the proof of the first part of Lemma 4.3 used only the unitarity of the matrix $u$, which holds for generators in $K\langle d\rangle$. Furthermore, given a 2-cocycle $c$ we can follow the proof of Lemma 4.4 define $\psi$ as in (4.5) and then show that $t_{j k}$ 's satisfy the relations defining $K\langle d\rangle$, so that one can deduce that $c$ is a coboundary (as noted above, this does not involve condition (4.4) ). Thus we obtain the following proposition.

Proposition 4.5. Let $d \in \mathbb{N}$. The second cohomology group for the Brown-Glockner-von Waldenfels algebra $K\langle d\rangle$ with trivial coefficients is trivial: $H^{2}\left(K\langle d\rangle,{ }_{\epsilon} \mathbb{C}_{\epsilon}\right)=0$. 
We are almost ready for the proof of the main result of this subsection; we still need to introduce the 2-cocycles which will lead to a basis of $H^{2}\left(U_{d}^{+}\right)$. Assume that $d \geq 3$.

Denote by $e_{j k}(j, k=1, \ldots, d)$ the canonical matrix units in $M_{d}(\mathbb{C})$. By Proposition 3.2. each $e_{j k}$ defines a Gaussian 1-cocycle $\eta_{j k}$ on $\operatorname{Pol}\left(U_{d}^{+}\right)$. Thus further, by Lemma 4.2, if $m, n, l \in\{1, \ldots, d\}$ (and we view $l$ as an arbitrary index depending on $m$ and $n$ ), then

$$
K_{m, n}=K\left(\eta_{e_{l m}}, \eta_{e_{l n}}\right)
$$

is a 2-cocycle. Moreover, for each $p \in\{1, \ldots, d-1\}$ define the matrix $V_{p} \in M_{d}(\mathbb{C})$ by inserting the matrix $V=\left(\begin{array}{ll}1 & i \\ 0 & 1\end{array}\right)$ into the $(p, p+1)$-block. More precisely, we have $\left(V_{p}\right)_{p, p}=1$, $\left(V_{p}\right)_{p, p+1}=i,\left(V_{p}\right)_{p+1, p+1}=1$ and all the other entries are 0 . Any such $V_{p}$ defines a Gaussian 1-cocycle $\eta_{V_{p}}$, and hence a 2-cocycle

$$
K_{p}:=K\left(\eta_{V_{p}}, \eta_{V_{p}}\right)
$$

on $\operatorname{Pol}\left(U_{d}^{+}\right)$.

Theorem 4.6. Let $d \in \mathbb{N}$. Then

$$
H^{2}\left(U_{d}^{+},{ }_{\epsilon} \mathbb{C}_{\epsilon}\right) \cong \mathbb{C}^{d^{2}-1} .
$$

Furthermore for $d \geq 2$ the set $\widetilde{Y}_{d}=\left\{\left[K_{m, n}\right]: m \neq n, m, n=1, \ldots, d\right\} \cup\left\{\left[K_{p}\right]: p=1, \ldots, d-1\right\}$ is a basis of $H^{2}\left(U_{d}^{+},{ }_{\epsilon} \mathbb{C}_{\epsilon}\right)$.

Proof. Define $\Delta: Z^{2}\left(U_{d}^{+}\right) \rightarrow M_{d}(C)$ by the formula

$$
\Delta(c)=\left(\sum_{p=1}^{d}\left(c\left(u_{p j}^{*} \otimes u_{p k}\right)-c\left(u_{k p}^{*} \otimes u_{j p}\right)\right)\right)_{j, k=1}^{d} .
$$

Observe that $\Delta\left(Z^{2}\left(U_{d}^{+}\right)\right) \subseteq \operatorname{sl}(d, \mathbb{C})$, where $\operatorname{sl}(d, \mathbb{C})$ denotes the space of $d \times d$ complex matrices with trace zero; indeed, for any $c \in Z^{2}\left(U_{d}^{+}\right)$

$$
\operatorname{Tr}(\Delta(c))=\sum_{j, p=1}^{d} c\left(u_{p j}^{*} \otimes u_{p j}\right)-\sum_{j, p=1}^{d} c\left(u_{j p}^{*} \otimes u_{j p}\right)=0 .
$$

Next we check that $\Delta\left(Z^{2}\left(U_{d}^{+}\right)\right)=\operatorname{sl}(d, C)$, i.e. that $\Delta$ is surjective. Assume then that $d \geq 2$, recall (4.6)-(4.7) and compute $(p \in\{1, \ldots, d-1\}, j, k, m, n \in\{1, \ldots, d\}$ and $m \neq n)$

$$
\begin{aligned}
\left(\Delta\left(K_{p}\right)\right)_{j k} & =\sum_{s=1}^{d}\left(\left\langle\eta_{p}\left(u_{s j}\right), \eta_{p}\left(u_{s k}\right)\right\rangle-\left\langle\eta_{p}\left(u_{k s}\right), \eta_{p}\left(u_{j s}\right)\right\rangle\right) \\
& =\left(V_{p}^{*} V_{p}-\left(\bar{V}_{p} V_{p}^{t}\right)^{t}\right)_{j k}=\left[V_{p}^{*}, V_{p}\right]_{j k}=\delta_{j p} \delta_{k p}-\delta_{j, p+1} \delta_{k, p+1}, \\
\left(\Delta\left(K_{m, n}\right)\right)_{j k} & =\sum_{s=1}^{d}\left(\left\langle\eta_{l m}\left(u_{s j}\right), \eta_{l n}\left(u_{s k}\right)\right\rangle-\left\langle\eta_{l m}\left(u_{k s}\right), \eta_{l n}\left(u_{j s}\right)\right\rangle\right) \\
& =\left[e_{l m}^{*} e_{l n}-\left(\bar{e}_{l m} e_{l n}^{t}\right)^{t}\right]_{j k}=\left[e_{m l} e_{l n}-e_{l n} e_{m l}\right]_{j k}=\left(e_{m n}\right)_{j k} .
\end{aligned}
$$

This shows that

$$
\Delta\left(K_{p}\right)=e_{p p}-e_{p+1, p+1}, \quad \Delta\left(K_{m, n}\right)=e_{m n},
$$

and surjectivity of $\Delta$ follows from the fact that the matrices appearing on the right-hand-side form together a basis for $\operatorname{sl}(d, C)$. 
Finally, we note that Lemma 4.4 shows that $\operatorname{ker} \Delta=B^{2}\left(U_{d}^{+}\right)$. Hence $\Delta$ induces a linear isomorphism from $H^{2}\left(U_{d}^{+}\right)=Z^{2}\left(U_{d}^{+}\right) / B^{2}\left(U_{d}^{+}\right)$to $\operatorname{sl}(d, \mathbb{C})$ and the conclusions of the theorem hold for $d \geq 2$.

The case $d=1$ is trivial.

4.2. Computing $H^{2}\left(O_{d}^{+}\right)$. The method presented in the last subsection provides an alternative, elementary proof of the fact that

$$
H^{2}\left(O_{d}^{+}\right) \cong \mathbb{C}^{\frac{d(d-1)}{2}}
$$

as proved by Collins, Härtel and Thom in [CHT, Theorem 3.2] (see also [Bic 1 , Proposition 6.4]). It also provides a basis of the space $H^{2}\left(O_{d}^{+}\right)$.

Comparing to the unitary case, for $O_{d}^{+}$the 'defect' map $\Delta$, which measures how far a cocycle is from being a coboundary, maps the space of 2-cocycles to $o(d)=o(d, \mathbb{C})$, the space of anti-symmetric complex $d \times d$ matrices. This difference comes from the fact that for $O_{d}^{+}$ we have the additional relation $u_{j k}^{*}=u_{j k}$. We sketch below the corresponding arguments.

Lemma 4.7. For every normalised $c \in Z^{2}\left(O_{d}^{+}\right)$we have for all $j, k \in\{1, \ldots, d\}$

$$
\sum_{p=1}^{d} c\left(u_{p j} \otimes u_{p k}\right)=\sum_{p=1}^{d} c\left(u_{j p} \otimes u_{k p}\right) .
$$

Proof. Apply $\partial c$ to $\sum_{s, p=1}^{d} u_{j s} \otimes u_{p s} \otimes u_{p k}$.

Lemma 4.8. A 2-cocycle $c \in Z^{2}\left(O_{d}^{+}\right)$is a coboundary if and only if for all $j, k \in\{1, \ldots, d\}$

$$
\sum_{p=1}^{d} c\left(u_{j p} \otimes u_{k p}\right)=\sum_{p=1}^{d} c\left(u_{k p} \otimes u_{j p}\right) .
$$

Proof. Assume (as we may) that $c$ is normalised.

$(\Rightarrow)$ Apply $-\partial \psi=c$ to $\sum_{p=1}^{d} u_{j p} \otimes u_{k p}$ and to $\sum_{p=1}^{d} u_{p j} \otimes u_{p k}$ and subtract the resulting equations.

$(\Leftarrow)$ Set $\psi\left(u_{j k}\right)=\frac{1}{2} \sum_{p=1}^{d} c\left(u_{p j} \otimes u_{p k}\right)$ (note that the two sums above coincide and define a symmetric matrix) and use Lemma 4.1 to prove that $\partial \psi=c$.

Let for the moment $d \geq 3$. For any $j, k \in\{1, \ldots, d\}$ consider the matrices

$$
Z_{j k}=e_{j k}-e_{k j}
$$

and the associated Gaussian 1-cocycles (see Proposition 3.7) $\eta_{Z_{j k}}$ on $O_{d}^{+}$. Then for any $m, n \in\{1, \ldots, d\}, m<n$, choose $l \in\{1, \ldots, d\}$ different from both $m$ and $n$, and define

$$
\widehat{K}_{m n}=K\left(\eta_{Z_{l m}}, \eta_{Z_{l n}}\right) \text {. }
$$

Note that according to Lemma 4.2, each $\widehat{K}_{m n}$ is a 2-cocycle.

Theorem 4.9. Let $d \in \mathbb{N}$. Then

$$
H^{2}\left(O_{d}^{+},{ }_{\epsilon} \mathbb{C}_{\epsilon}\right) \cong \mathbb{C}^{\frac{d(d-1)}{2}}
$$

Furthermore for $d \geq 3$ the set $\tilde{Y}_{d}=\left\{\left[\widehat{K}_{m, n}\right]: m<n, m, n=1, \ldots, d\right\}$ is a basis of $H^{2}\left(O_{d}^{+},{ }_{\epsilon} \mathbb{C}_{\epsilon}\right)$. 
Proof. We just sketch the proof, analogous to that of Theorem 4.6, with the map $\Delta_{O}$ : $Z^{2}\left(O_{d}^{+}\right) \rightarrow M_{d}$ given by

$$
\Delta_{O}(c)=\left(\sum_{p=1}^{d}\left(c\left(u_{j p} \otimes u_{k p}\right)-c\left(u_{k p} \otimes u_{j p}\right)\right)\right)_{j, k=1}^{d},
$$

which clearly yields an anti-symmetric matrix. If $d \geq 3$ then we check that

$$
\begin{aligned}
\Delta_{O}\left(\widehat{K}_{m n}\right) & =\left(\sum_{p=1}^{d}\left(\left\langle\eta_{Z_{l m}}\left(u_{j p}\right), \eta_{Z_{l n}}\left(u_{k p}\right)\right\rangle-\left\langle\eta_{Z_{l m}}\left(u_{k p}\right), \eta_{Z_{l n}}\left(u_{j p}\right)\right\rangle\right)_{j, k=1}^{d}\right. \\
& =\bar{Z}_{l m} Z_{l n}^{t}-\left(\bar{Z}_{l m} Z_{l n}^{t}\right)^{t}=\left(e_{l m}-e_{m l}\right)\left(e_{n l}-e_{l n}\right)-\left(e_{l n}-e_{n l}\right)\left(e_{m l}-e_{l m}\right) \\
& =e_{m n}-e_{n m}=Z_{m n} .
\end{aligned}
$$

Since the set of matrices $Z_{m n}$ defines a basis of $o(d)$, the map $\Delta_{O}$ is surjective. Finally, by Lemma 4.8, $B^{2}\left(O_{d}^{+}\right)=\Delta_{O}\left(Z^{2}\left(O_{d}^{+}\right)\right)$, and thus $\Delta_{O}$ establishes the homomorphism between $H^{2}\left(O_{d}^{+}\right)=Z^{2}\left(O_{d}^{+}\right) /$ker $\Delta_{O}$ and $o(d)$.

Now if $d=2$ it suffices to exhibit a 2-cocycle $c \in Z^{2}\left(O_{2}^{+}\right)$such that $\Delta_{O}(c) \neq 0$. To this end it suffices to consider matrices $Z_{1}=\left(\begin{array}{cc}1 & 0 \\ 0 & -1\end{array}\right)$ and $Z_{2}=\left(\begin{array}{ll}0 & 1 \\ 1 & 0\end{array}\right)$, corresponding antiGaussian cocycles (see Proposition [3.7) $\eta_{Z_{1}}$ and $\eta_{Z_{2}}$, and the 2-cocycle $K\left(\eta_{Z_{1}}, \eta_{Z_{2}}\right)$ defined via Lemma 4.2, Then one can check that

$$
\Delta_{O}\left(K\left(\eta_{Z_{1}}, \eta_{Z_{2}}\right)\right)=\left(\begin{array}{cc}
0 & 2 \\
-2 & 0
\end{array}\right)
$$

and the proof is finished (the case $d=1$ is trivial).

\section{ACKNOWLEDGEMENTS}

BD would like to thank the support of Mathematics Research Unit, University of Oulu in the years 2016-2017, when a part of the work was done. UF was supported by the French "Investissements d'Avenir" program, project ISITE-BFC (contract ANR-15-IDEX-03). AK was supported by the National Science Centre grant SONATA 2016/21/D/ST1/03010. AS was partially supported by the NCN (National Science Centre) grant 2014/14/E/ST1/00525. UF and AS acknowledge support by the French MAEDI and MENESR and by the Polish MNiSW through the Polonium programme. We thank Julien Bichon and Malte Gerhold for useful comments.

\section{REFERENCES}

[Acc] L. Accardi, Quantum probability: an historical survey, in Probability on algebraic structures (Gainesville, FL, 1999), 145-159, Contemp. Math., 261, Amer. Math. Soc., Providence, RI, 2000.

[ASvW] L. Accardi, M. Schürmann and W. von Waldenfels, Quantum independent increment processes on superalgebras, Math. Z. 198 (1988), no. 4, 451-477.

[App 1 D. Applebaum, "Lévy processes and stochastic calculus," Cambridge Studies in Advanced Mathematics, 116. Cambridge University Press, Cambridge, 2009.

[App 2$]$ D. Applebaum, "Probability on compact Lie groups," Probability Theory and Stochastic Modelling, 70. Springer, 2014. 
[Ara] H. Araki, Factorizable representation of current algebra. Non commutative extension of the LévyKhinchin formula and cohomology of a solvable group with values in a Hilbert space, Publ. Res. Inst. Math. Sci. 5 1969/1970, 361-422.

$\left[\mathrm{Ban}_{1}\right]$ T. Banica, Théorie des représentations du groupe quantique compact libre $O(n), C . R$. Acad. Sci. Paris Sér. I Math. 322 (1996), no. 3, 241-244.

$\left[\mathrm{Ban}_{2}\right] \quad$ T. Banica, Le groupe quantique compact libre $U(n)$, Comm. Math. Phys. 190 (1997), no. 1, $143-172$.

$[\mathrm{BaV}]$ T. Banica and R. Vergnioux, Invariants of the half-liberated orthogonal group Annales de l'institut Fourier 60 (2010), no. 6, 2137-2164.

[Bic $\left.{ }_{1}\right]$ J. Bichon, Hochschild homology of Hopf algebras and free Yetter-Drinfeld resolutions of the counit, Compos. Math. 149 (2013), no. 4, 658-678.

[Bic 2 J. Bichon, Homological invariants for discrete quantum groups, Notes for a mini-course given at the conference "Topological quantum groups and harmonic analysis", May 15-19, 2017 at Seoul National University, http://www.math.snu.ac.kr/ hhlee/Bichon-Notes.pdf

[BFG] J. Bichon, U. Franz and M. Gerhold, Homological properties of quantum permutation algebras, New York Journal of Mathematics 23 (2017), 1697-1721

[Bro] L. G. Brown, Ext of certain free product C ${ }^{*}$-algebras, J. Operator Theory 6 (1981), no. 1, $135-141$.

[CFK] F. Cipriani, U. Franz and A. Kula, Symmetries of Lévy processes on compact quantum groups, their Markov semigroups and potential theory, J. Funct. Anal. 266 (2014), no. 5, 2789-2844.

[CHT] B. Collins, J. Härtel and A. Thom, Homology of free quantum groups, C. R. Math. Acad. Sci. Paris, 347 (2009), 271-276.

[dRi] An de Rijdt, Monoidal equivalence of compact quantum groups, PhD thesis, Katholieke Universiteit Leuven, 2007.

[DFKS] B. Das, U. Franz, A. Kula and A.Skalski, One-to-one correspondence between generating functionals and cocycles on quantum groups in presence of symmetry, Math. Z. 281 (2015), no. 3-4, 949-965.

[DKSS] M. Daws, P. Kasprzak, A. Skalski and P. Sołtan, Closed quantum subgroups of locally compact quantum groups, Adv. Math. 231 (2012), 3473-3501.

[DiK] M. Dijkhuizen and T. Koornwinder, CQG algebras — a direct algebraic approach to compact quantum groups, Lett. Math. Phys. 32 (1994) no. 4, 315-330.

[FKS] U. Franz, A. Kula and A. Skalski, Lévy processes on quantum permutation groups, in Noncommutative analysis, operator theory and applications, 193-259, Oper. Theory Adv. Appl., 252, Linear Oper. Linear Syst., Birkhäuser/Springer, 2016.

[FGT] U. Franz, M. Gerhold and A. Thom, On the Lévy-Khinchin decomposition of generating functionals. Comm. Stoch. Anal. 9 (2015), no. 4 529-544.

[FS] U. Franz and R.Schott, Stochastic Processes and Operator Calculus on Quantum Groups, Kluwer Academic Publishers, Dordrecht, 1999.

$[\mathrm{GvW}$ P. Glockner and W. von Waldenfels, The relations of the noncommutative coefficient algebra of the unitary group, in Quantum probability and applications, IV (Rome, 1987), 182-220, Lecture Notes in Math., 1396, Springer, Berlin, 1989.

[Kye] D. Kyed, A cohomological description of property (T) for quantum groups, J. Funct. Anal. 261 (2011), no. 6, 1469-1493.

[Pod] P. Podleś, Symmetries of quantum spaces. Subgroups and quotient spaces of quantum SU(2) and SO(3) groups, Comm. Math. Phys., 170(1995), no. 1, 1-20.

[Sch $\left.{ }_{1}\right] \quad$ M. Schürmann, Gaussian states on bialgebras, in Quantum Probability and Applications V, Lecture Notes in Math. 1442 (1990) 347-367.

[Sch $\mathrm{Sch}_{2} \quad$ M. Schürmann, "White noise on bialgebras," Lecture Notes in Math. 1544. Springer-Verlag, Berlin, 1993.

[Sch 3 M. Schürmann, Non-commutative probability on algebraic structures, in Probability measures on groups and related structures, XI (Oberwolfach, 1994), 332-356, World Sci. Publ., River Edge, NJ, 1995.

[ScS] M. Schürmann and M. Skeide, Infinitesimal generators on the quantum group $\mathrm{SU}_{q}(2)$, Infin. Dimens. Anal. Quantum Probab. Relat. Top. 1 (1998), no. 1, 573-598.

[Ske $\left.{ }_{1}\right]$ M. Skeide, The Lévy-Khintchine formula for the quantum group $S U_{q}(2)$, PhD thesis, Heidelberg, 1994, available at http://web.unimol.it/skeide/.

[Ske ${ }_{2}$ M. Skeide, Infinitesimal generators on the quantum group $S U_{q}(2)$ in the classical and anti-classical limit. Open Syst. Inf. Dyn. 6 (1999), no. 4, 375414. 
[Ske 3 ] M. Skeide, Hunt's formula on $S U_{q}(2)$; a unified view. Open Syst. Inf. Dyn. 6 (1999), 1-27.

[Str] R.F. Streater, Classical and quantum probability, J. Math. Phys. 41 (2000), no. 6, 3556-3603.

[Tim] T. Timmermann, "An invitation to quantum groups and duality.From Hopf algebras to multiplicative unitaries and beyond," EMS Textbooks in Mathematics. European Mathematical Society (EMS), Zürich, 2008.

[VD] A. Van Daele, Dual pairs of Hopf *-algebras, Bull. LMS 25 (1993), 209-230.

[VDW] A. Van Daele and S. Wang, Universal quantum groups, Int. J. Math. 7 (1996), no. 2, 255-263.

[Wo1] S.L. Woronowicz, Compact matrix pseudogroups, Comm. Math. Phys. 111 (1987) no. 4, 613-665.

$\left[\mathrm{Wo}_{2}\right] \quad$ S.L. Woronowicz, Tannaka-Krein duality for compact matrix pseudogroups. Twisted SU $(N)$ groups, Invent. Math., 93(1988), no. 135-176.

[Wo3] S.L. Woronowicz, Compact quantum groups, in "Symétries Quantiques," Proceedings, Les Houches 1995, eds. A. Connes, K. Gawedzki \&6 J. Zinn-Justin, North-Holland, Amsterdam 1998, pp. 845-884.

Instytut Matematyczny, Uniwersytet WrocŁaWski, Pl.Grunwaldzki 2/4, 50-384 WrocŁaW, POLAND

E-mail address: biswarup.das@math.uni.wroc.pl

Laboratoire de mathématiques de Besançon, Université de Bourgogne Franche-Comté, 16, Route de Gray, 25030 BesanÇOn CEDEX, France

E-mail address: uwe.franz@univ-fcomte.fr

$U R L:$ http://lmb.univ-fcomte.fr/uwe-franz

Instytut Matematyczny, Uniwersytet WrocŁawski, Pl.Grunwaldzki 2/4, 50-384 WrocŁaW, POLAND

E-mail address: Anna.Kula@math.uni.wroc.pl

Institute of Mathematics of the Polish ACAdemy of Sciences, ul. Śniadeckich 8, 00-656 WARszaWA, POLAND

E-mail address: a.skalski@impan.pl 